\title{
KELT-23Ab: A Hot Jupiter Transiting a Near-solar Twin Close to the TESS and JWST Continuous Viewing Zones
}

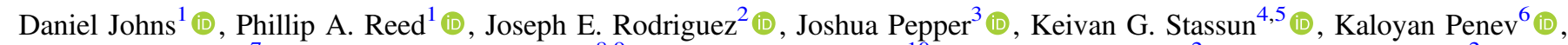

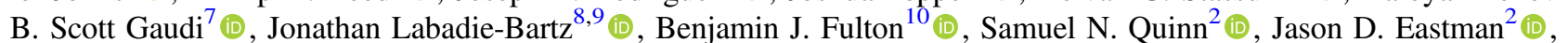
David R. Ciardi ${ }^{10}$, Lea Hirsch ${ }^{11}$ (i), Daniel J. Stevens ${ }^{12,13}$ (1), Catherine P. Stevens ${ }^{14}$, Thomas E. Oberst ${ }^{14}$, David H. Cohen ${ }^{15}$, Eric L. N. Jensen ${ }^{15}$ (1), Paul Benni ${ }^{16}$, Steven Villanueva, Jr. ${ }^{7}$ (), Gabriel Murawski ${ }^{17}$, Allyson Bieryla ${ }^{2}$ (1), David W. Latham ${ }^{2}$ (1) Siegfried Vanaverbeke ${ }^{18}$, Franky Dubois ${ }^{18}$, Steve Rau ${ }^{18}$, Ludwig Logie ${ }^{18}$, Ryan F. Rauenzahn ${ }^{1}$, Robert A. Wittenmyer ${ }^{19}$ (1), Roberto Zambelli $^{20}$, Daniel Bayliss ${ }^{21,22}$ (1) , Thomas G. Beatty $^{13,23}$ (1), Karen A. Collins ${ }^{2}$ (1) , Knicole D. Colón ${ }^{24}$ (1) , Ivan A. Curtis ${ }^{25}$, Phil Evans ${ }^{26}$, Joao Gregorio ${ }^{27}$, David James ${ }^{28}$ (1), D. L. Depoy ${ }^{29,30}$, Marshall C. Johnson ${ }^{7}$ (1) Michael D. Joner ${ }^{31}$,

David H. Kasper ${ }^{32}$, Somayeh Khakpash ${ }^{3}$ (10), John F. Kielkopf ${ }^{33}$, Rudolf B. Kuhn ${ }^{34,35}$, Michael B. Lund ${ }^{4,10}$ (D), Mark Manner ${ }^{36}$ (D), Jennifer L. Marshall ${ }^{29,30}$ (1) , Kim K. McLeod ${ }^{37}$ (1), Matthew T. Penny ${ }^{7}$ (1) , Howard Relles ${ }^{2}$, Robert J. Siverd ${ }^{4}$ (1),

Denise C. Stephens ${ }^{31}$, Chris Stockdale ${ }^{38}$ (1), Thiam-Guan $\operatorname{Tan}^{39}$ (D) , Mark Trueblood $^{40}$, Pat Trueblood ${ }^{40}$, and Xinyu Yao ${ }^{3}$ (1)

${ }^{1}$ Department of Physical Sciences, Kutztown University, Kutztown, PA 19530, USA

${ }^{2}$ Center for Astrophysics | Harvard \& Smithsonian, 60 Garden Steet, Cambridge, MA 02138, USA

${ }^{3}$ Department of Physics, Lehigh University, 16 Memorial Drive East, Bethlehem, PA, 18015, USA

${ }^{4}$ Department of Physics and Astronomy, Vanderbilt University, Nashville, TN 37235, USA

${ }^{5}$ Department of Physics, Fisk University, 1000 17th Avenue North, Nashville, TN 37208, USA

${ }^{6}$ Department of Physics, The University of Texas at Dallas, 800 West Campbell Road, Richardson, TX 75080-3021 USA

7 Department of Astronomy, The Ohio State University, 140 West 18th Avenue, Columbus, OH 43210, USA

${ }^{8}$ Instituto de Astronomia, Geofísica e Ciências Atmosféricas, Universidade de São Paulo, Rua do Matão 1226, Cidade Universitária, 05508-900, São Paulo, SP, Brazil

Department of Physics \& Astronomy, University of Delaware, Newark, DE 19716, USA

${ }^{10}$ Caltech-IPAC/NExScI, Caltech, Pasadena, CA 91125 USA

${ }^{11}$ Kavli Institute for Particle Astrophysics and Cosmology, Stanford University, Stanford, CA 94305, USA

${ }^{12}$ Department of Astronomy \& Astrophysics, The Pennsylvania State University, 525 Davey Lab, University Park, PA 16802, USA

${ }^{13}$ Center for Exoplanets and Habitable Worlds, The Pennsylvania State University, 525 Davey Lab, University Park, PA 16802, USA

${ }^{14}$ Department of Physics, Westminster College, New Wilmington, PA 16172, USA

${ }^{15}$ Department of Physics \& Astronomy, Swarthmore College, Swarthmore, PA 19081, USA

${ }^{6}$ Acton Sky Portal (private observatory), Acton, MA 01720, USA

${ }^{17}$ Gabriel Murawski Private Observatory, Suwalki, Poland

18 AstroLAB IRIS, Provinciaal Domein De Palingbeek, Verbrandemolenstraat 5, B-8902 Zillebeke, Ieper, Belgium

${ }^{19}$ University of Southern Queensland, Centre for Astrophysics, West Street, Toowoomba, QLD 4350 Australia

${ }^{20}$ Società Astronomica Lunae, Italy

${ }^{21}$ Research School of Astronomy and Astrophysics, Mount Stromlo Observatory, Australian National University, Cotter Road, Weston, ACT, 2611, Australia

${ }^{22}$ Department of Physics, University of Warwick, Gibbet Hill Road, Coventry, CV4 7AL, UK

${ }^{23}$ Department of Astronomy and Steward Observatory, University of Arizona, Tucson, AZ 85721, USA

${ }^{24}$ NASA Goddard Space Flight Center, Exoplanets and Stellar Astrophysics Laboratory (Code 667), Greenbelt, MD 20771, USA

${ }^{25}$ Ivan Curtis Private Observatory, Adelaide, Australia

${ }^{26}$ El Sauce Observatory, Chile

${ }^{27}$ Atalaia Group \& CROW Observatory, Portalegre, Portugal

${ }^{28}$ Event Horizon Telescope, Center for Astrophysics | Harvard \& Smithsonian, MS-42, 60 Garden Street, Cambridge, MA 02138, USA

${ }^{29}$ George P. and Cynthia Woods Mitchell Institute for Fundamental Physics and Astronomy, Texas A\&M University, College Station, TX77843 USA

${ }^{30}$ Department of Physics and Astronomy, Texas A\&M university, College Station, TX 77843 USA

${ }^{31}$ Department of Physics and Astronomy, Brigham Young University, Provo, UT 84602, USA

${ }^{32}$ Department of Physics \& Astronomy, University of Wyoming, 1000 E University Avenue, Dept 3905, Laramie, WY 82071, USA

33 Department of Physics and Astronomy, University of Louisville, Louisville, KY 40292 USA

${ }^{34}$ South African Astronomical Observatory, PO Box 9, Observatory, 7935, Cape Town, South Africa

${ }^{35}$ Southern African Large Telescope, PO Box 9, Observatory, 7935, Cape Town, South Africa

${ }^{36}$ Spot Observatory, Nashville, TN 37206, USA

${ }^{37}$ Department of Astronomy, Wellesley College, Wellesley, MA 02481, USA

${ }^{38}$ Hazelwood Observatory, Churchill, Victoria, Australia

${ }^{39}$ Perth Exoplanet Survey Telescope, Perth, Australia

${ }^{40}$ Winer Observatory, PO Box 797, Sonoita, AZ 85637, USA

Received 2019 February 27; revised 2019 May 21; accepted 2019 May 23; published 2019 July 24

\begin{abstract}
We announce the discovery of KELT-23Ab, a hot Jupiter transiting the relatively bright $(V=10.3)$ star BD+66 911 (TYC 4187-996-1), and characterize the system using follow-up photometry and spectroscopy. A global fit to the system yields host-star properties of $T_{\text {eff }}=5900 \pm 49 \mathrm{~K}, M_{*}=0.945_{-0.054}^{+0.060} M_{\odot}, R_{*}=0.995 \pm 0.015 R_{\odot}$, $L_{*}=1.082_{-0.048}^{+0.051} L_{\odot}, \log g_{\star}=4.418_{-0.025}^{+0.026}(\mathrm{cgs})$, and $[\mathrm{Fe} / \mathrm{H}]=-0.105 \pm 0.077$. KELT-23Ab is a hot Jupiter with a mass of $M_{P}=0.938_{-0.042}^{+0.045} M_{\mathrm{J}}$, radius of $R_{P}=1.322 \pm 0.025 R_{\mathrm{J}}$, and density of $\rho_{P}=0.504_{-0.035}^{+0.038} \mathrm{~g} \mathrm{~cm}^{-3}$. Intense insolation flux from the star has likely caused KELT-23Ab to become inflated. The time of inferior conjunction is $T_{0}=2458149.40776 \pm 0.00091 \mathrm{BJD}_{\mathrm{TDB}}$ and the orbital period is $P=2.255353_{-0.000030}^{+0.00031}$ days. There is strong evidence that KELT-23A is a member of a long-period binary star system with a less luminous companion, and due to tidal interactions, the planet is likely to spiral into its host within roughly a gigayear. This system has one of the highest positive ecliptic latitudes of all transiting planet hosts known to date, placing it near
\end{abstract}


the Transiting Planet Survey Satellite and James Webb Space Telescope continuous viewing zones. Thus we expect it to be an excellent candidate for long-term monitoring and follow up with these facilities.

Key words: methods: observational - planets and satellites: detection - planets and satellites: gaseous planets techniques: photometric - techniques: radial velocities - techniques: spectroscopic

\section{Introduction}

The first known transiting exoplanet, HD 209458b (Charbonneau et al. 2000; Henry et al. 2000), is now but one of over 3000 confirmed exoplanets. Most of these planets were discovered by the Kepler mission (Borucki et al. 2010; Howell et al. 2014; Coughlin et al. 2016), and the first of many new discoveries are now coming from the Transiting Exoplanet Survey Satellite (TESS; Ricker et al. 2015; Huang et al. 2018; Vanderspek et al. 2019). The short-period, massive planet population known as hot Jupiters produces signals that are large enough to be detected from ground-based transit surveys such as the Trans-Atlantic Exoplanet Survey (Alonso et al. 2004), the XO project (McCullough et al. 2005), the Wide Angle Search for Planets (WASP; Pollacco et al. 2006), the Hungarian Automated Telescope Network (Bakos et al. 2004), the Qatar Exoplanet Survey (Alsubai et al. 2013), the NextGeneration Transit Survey (Wheatley et al. 2013), the Multisite All Sky Camera (Talens et al. 2017), and the Kilodegree Extremely Little Telescope (KELT; Pepper et al. 2003, 2007, 2012).

The majority of the known Kepler planets orbit faint stars, but have relatively long periods compared to the transiting planets found by ground-based surveys due to the long duration and continuous observations of Kepler's prime survey. Indeed, ground-based surveys are typically only sensitive to planets with periods of less than roughly 10 days due primarily to the effects of weather, the diurnal cycle, and the atmosphere, resulting in a noncontinuous cadence and poor sensitivity to longer planets, but find planets around stars that are much brighter than Kepler due to their smaller apertures and ability to monitor much larger regions of the sky. One such survey, KELT, ${ }^{41}$ is a pair of telescopes situated in the northern and southern hemispheres, in Arizona (KELT-North) and South Africa (KELT-South), respectively. KELT-North and KELTSouth each have apertures $42 \mathrm{~mm}$ in diameter with a $26^{\circ} \times 26^{\circ}$ field of view and a $23^{\prime \prime}$ pixel scale. KELT-North and KELTSouth began collecting data in 2005 and 2009, respectively. Between them, they survey roughly $61 \%$ of the sky at $10-20$ minutes cadences with about $1 \%$ photometric precision down to $V \sim 11$. KELT is most sensitive to giant planets transiting stars between the 8th and 11th magnitude in the $V$ band, a range that is often missed by radial velocity surveys and many other transit surveys, because either the hosts are too faint and numerous (for RV), or because stars at the brighter end of this magnitude range are saturated for ground-based surveys with larger apertures than KELT. This range implies that KELT is biased toward detecting inflated, giant planets (Gaudi 2005) around hot, luminous stars (Bieryla et al. 2015). Since the targets selected by KELT are relatively bright, they present a valuable opportunity for atmospheric characterization. KELT has discovered over 20 exoplanets, the latest being KELT-21b (Johnson et al. 2018a) from KELT-North and KELT-22Ab (Labadie-Bartz et al. 2019) from KELT-South.

\footnotetext{
${ }^{41}$ https://keltsurvey.org
}

The inflated giant planets sought by KELT are hot Jupiters. They typically have masses greater than $0.25 M_{\mathrm{J}}$, radii between 1 and $2 R_{\mathrm{J}}$, and periods less than 10 days. They present a unique opportunity to study the evolution and formation of exoplanetary systems. Hot Jupiters are fairly easy to detect, however Wright et al. (2012) and Fressin et al. (2013) estimate that only around $1 \%$ of Sun-like stars host hot Jupiters, making them relatively rare compared to smaller planets. The frequency and depth of photometric transits of the hot Jupiters make them optimal for detection by the abovementioned surveys. KELT plays an important role discovering and confirming some of the most promising hot Jupiters for follow-up observations by the Hubble Space Telescope (HST), Spitzer Space Telescope (see Beatty et al. 2018), and the James Webb Space Telescope (JWST).

Gas giants also present a unique opportunity to study atmospheric characteristics in planets outside of our solar system. Transmission spectroscopy is the illumination through the planet's atmosphere by the host star during a transit. This effect allows us to study the atmospheric composition, wind speeds, haze, rotation rate, etc. (Charbonneau et al. 2002; Brogi et al. 2016; Sing et al. 2016). In addition to transmission spectroscopy, secondary eclipses give insight into the planet's albedo and thermal emission in the near-infrared (Garhart et al. 2019).

Here we report the discovery of KELT-23Ab, a hot Jupiter orbiting a Sun-like star. KELT-23A is a bright $(V=10.25)$, near-solar metallicity $([\mathrm{Fe} / \mathrm{H}]=-0.105)$, main-sequence, G2type host whose composition may give insight into planetary formation processes around Sun-like stars. Meléndez et al. (2009) found that the Sun's composition has a distinguishing abundance pattern that is not shared by solar analogs known to host hot Jupiters. More recently, Bedell et al. (2018) analyzed the abundance ratios of Sun-like exoplanet hosts within the solar neighborhood and found that a majority of them have similar abundance patterns. A differential abundance analysis may give clues as to why the solar system does not host a hot Jupiter, yet KELT-23A does. Our analysis suggests that KELT$23 \mathrm{Ab}$ is on a decaying orbit and is spiraling in toward the host star, a circumstance that could provide an opportunity to better understand the late stages of giant planet orbital evolution around a Sun-like star.

In Section 2 we describe the initial discovery light curve, follow-up photometry and spectroscopy, and adaptive optics (AO) observations. In Section 3 we use follow-up observations to characterize the host star. In Section 4 we fit a global model to the planetary system to measure the planet's parameters. We also search for transit timing variations (TTVs) and consider the likelihood of a wide binary companion to KELT-23A. We conclude that the planetary host is the brighter of the two stars and have therefore given it the appropriate designation (" $\mathrm{A}$ ") in its name. Finally, in Sections 5 and 6 we rule out the possibility of a false-positive scenario and discuss the tidal evolution of the system, stellar abundances, and future observations. 


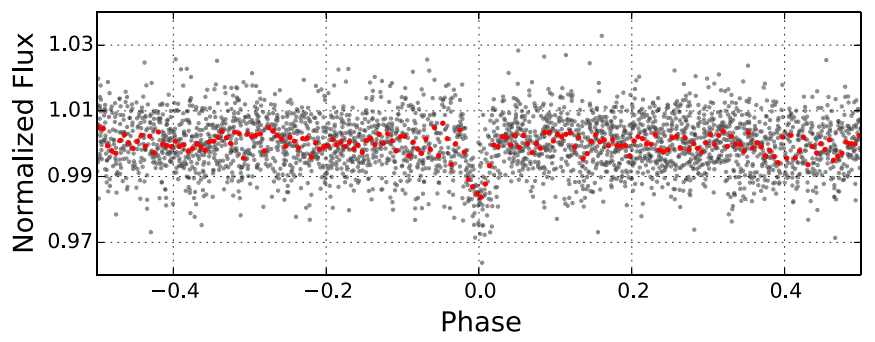

Figure 1. Discovery light curve of KELT-23A containing 3308 observations from the KELT-North telescope phase-folded on the discovery period of 2.2552347 days. The red points are the data binned on an 18-minute timescale.

\section{Discovery and Follow-up Observations}

\subsection{KELT-North Observations and Photometry}

KELT-23A is located in KELT-North survey field 22, a $26^{\circ} \times 26^{\circ}$ region centered at $\left(\alpha_{\mathrm{J} 2000}=16^{\mathrm{h}} 03^{\mathrm{m}} 12^{\mathrm{s}} .0, \delta_{\mathrm{J} 2000}=\right.$ $+57^{\circ} 00^{\prime} 00^{\prime \prime} 0$ ). We observed the field a total of 3308 times from 2012 February to 2017 August. TYC 4187-996-1 was identified as a transit candidate within the field at $\alpha=15^{\mathrm{h}} 28^{\mathrm{m}} 35^{\mathrm{s}}$. $1926, \delta=+66^{\circ} 21^{\prime} 31^{\prime \prime} .544$. The initial discovery light curve, shown in Figure 1, contains a significant box-least-squares (Kovács et al. 2002) signal with a period of 2.2552375 days, a transit duration of $1.80 \mathrm{hr}$, and a transit depth of $12.8 \mathrm{mmag}$. The KELT image analysis and light-curve production pipeline is described in detail in Siverd et al. (2012). Table 1 shows photometric and astrometric properties of KELT-23A.

\subsection{Photometric Time-series Follow-up}

The KELT-Follow-Up Network (FUN) is a collaboration of around 60 observatories spread throughout the northern and southern hemispheres. ${ }^{42}$ The KELT-FUN follow-up photometry is used to better constrain the transit parameters, eliminate false positives, and determine accurate ephemerides. KELTFUN members plan photometric follow-up observations through the use of TAPIR (Jensen 2013), a web-based transit prediction calculator. KELT-FUN members generally use the AstroImageJ (AIJ) package (Collins \& Kielkopf 2013; Collins et al. 2017) $)^{43}$ to reduce and perform a preliminary analysis of their light curves. We obtained 11 follow-up transits between 2018 January 26 and July 3 from the observatories listed in Table 2. The specifications listed in Table 2 are taken from Collins et al. (2018), which is the most current listing of KELTFUN instrument specifications. The follow-up light curves span the Johnson $B V R I$ and Sloan $g^{\prime} r^{\prime} i^{\prime} z^{\prime}$ filter sets. As part of the follow-up confirmation process the transits in different bandpasses were checked for chromatic variations. No chromatic variations were found. The individual light curves are shown in Figure 2.

The KELT-FUN light curves were detrended by various parameters such as airmass, FWHM, X position, Y position, sky brightness, and the total comparison of star counts. To determine the best set of detrending parameters we used the Bayesian information criterion (BIC) of a preliminary fit to the detrended data. Detrending parameters that produced a drop in

\footnotetext{
42 A more complete listing of KELT-FUN observatories can be found at https://keltsurvey.org/.

43 https://www.astro.louisville.edu/software/astroimagej/
}

Table 1

Literature Properties for KELT-23A

\begin{tabular}{|c|c|c|c|}
\hline Other Names & $\begin{array}{l}\text { BD+66 } 911 \\
\text { TYC 4187-996-1 } \\
\text { TIC 458478250 }\end{array}$ & & \\
\hline Parameter & Description & Value & References \\
\hline$\alpha_{\mathbf{J} 2000}$ & Right ascension (R.A.) & $15^{\mathrm{h}} 28^{\mathrm{m}} 35^{\mathrm{s}} .1926$ & 1 \\
\hline$\delta_{\mathbf{J} 2000}$ & Declination (decl.) & $+66^{\circ} 21^{\prime} 31^{\prime \prime} .544$ & 1 \\
\hline$\beta_{\mathbf{J} 2000}$ & Ecliptic latitude & $+75^{\circ} 6^{\prime} 43^{\prime \prime} .21$ & 1 \\
\hline$\lambda_{\mathrm{J} 2000}$ & Ecliptic longitude & $163^{\circ} 17^{\prime} 10^{\prime \prime} .38$ & 1 \\
\hline$B_{\mathrm{T}}$ & Tycho $B_{\mathrm{T}}$ mag. & $11.029 \pm 0.049$ & 2 \\
\hline$V_{\mathrm{T}}$ & Tycho $V_{\mathrm{T}}$ mag. & $10.376 \pm 0.039$ & 2 \\
\hline$B$ & APASS Johnson $B$ mag. & $10.973 \pm 0.021$ & 3 \\
\hline$V$ & APASS Johnson $V$ mag. & $10.253 \pm 0.024$ & 3 \\
\hline$g^{\prime}$ & APASS Sloan $g^{\prime}$ mag. & $10.647 \pm 0.026$ & 3 \\
\hline$r^{\prime}$ & APASS Sloan $r^{\prime}$ mag. & $10.114 \pm 0.080$ & 3 \\
\hline$i^{\prime}$ & APASS Sloan $i^{\prime}$ mag. & $9.980 \pm 0.070$ & 3 \\
\hline$J$ & 2MASS $J$ mag. & $9.208 \pm 0.032$ & 4 \\
\hline$H$ & 2MASS $H$ mag. & $\geqslant 8.951^{*}$ & 4 \\
\hline$K$ & 2MASS $K$ mag. & $\geqslant 8.904^{*}$ & 4 \\
\hline WISE1 & WISE1 mag. & $8.746 \pm 0.022$ & 5 \\
\hline WISE2 & WISE2 mag. & $8.778 \pm 0.020$ & 5 \\
\hline WISE3 & WISE3 mag. & $8.778 \pm 0.019$ & 5 \\
\hline WISE4 & WISE4 mag. & $8.672 \pm 0.189$ & 5 \\
\hline$\mu_{\alpha}$ & $\begin{array}{l}\text { Gaia DR2 proper motion } \\
\text { in R.A. }\left(\text { mas } \mathrm{yr}^{-1}\right)\end{array}$ & $0.434 \pm 0.039$ & 1 \\
\hline$\mu_{\delta}$ & $\begin{array}{l}\text { Gaia DR2 proper motion } \\
\text { in decl. }\left(\text { mas } \mathrm{yr}^{-1}\right)\end{array}$ & $-12.217 \pm 0.041$ & 1 \\
\hline$\Pi$ & Gaia DR2 parallax (mas) & $7.973 \pm 0.021$ & $1 \dagger$ \\
\hline$R V$ & $\begin{array}{l}\text { Systemic radial } \\
\text { velocity }\left(\mathrm{km} \mathrm{s}^{-1}\right)\end{array}$ & $-15.224 \pm 0.10$ & Section 2.3 \\
\hline$v \sin i_{\star}$ & $\begin{array}{l}\text { Stellar rotational } \\
\text { velocity }\left(\mathrm{km} \mathrm{s}^{-1}\right)\end{array}$ & $2.42 \pm 0.50$ & Section 3.1 \\
\hline Sp. Type & Spectral type & G2V & Section 3 \\
\hline Age & Age (Gyr) & $6.3_{-3.2}^{+3.5}$ & Section 3.3 \\
\hline$d_{\star}$ & Distance $(\mathrm{pc})$ & $125.42 \pm 0.35$ & $1 \dagger$ \\
\hline$A_{V}$ & Visual extinction (mag) & $0.075 \pm 0.015$ & Section 3.2 \\
\hline$U^{\dagger \dagger}$ & Space motion $\left(\mathrm{km} \mathrm{s}^{-1}\right)$ & $17.32 \pm 0.03$ & Section 4.2 \\
\hline$V$ & Space motion $\left(\mathrm{km} \mathrm{s}^{-1}\right)$ & $0.95 \pm 0.07$ & Section 4.2 \\
\hline$W$ & Space motion $\left(\mathrm{km} \mathrm{s}^{-1}\right)$ & $-0.90 \pm 0.07$ & Section 4.2 \\
\hline
\end{tabular}

Note. All photometric apertures are blended with the partner within 4". 5 . References are: (1) Gaia Collaboration et al. (2018) Gaia DR2 http://gea.esac. esa.int/archive/; (2) Høg et al. (2000); (3) Henden et al. (2015); (4) Cutri et al. (2003); (5) Cutri et al. (2012). *Two Micron All Sky Survey (2MASS) magnitude contains the "U" quality flag. $†$ Gaia DR2 parallax and distance after correcting for the systematic offset of -0.082-mas as described in Stassun \& Torres (2018). $\dagger \dagger-U$ is taken to be positive in the direction of the galactic center.

BIC of 10 or greater were selected for the global fit (Section 4.1). These free parameters can be found in Table 2. In all cases, airmass was selected as a detrending parameter due to the pervasive airmass-dependent systematic effects seen in differential photometry. Other parameters such as the $\mathrm{X}$ and $\mathrm{Y}$ target pixel position were evaluated as they can induce trends in the light curve due to flat fielding. Observatories that perform a meridian flip during observations additionally have the option of detrending by meridian flip in AIJ as a meridian flip can cause a shift in the position of the target. Meridian flip detrending was not found to be necessary for any KELT-FUN light curves in the current analysis. 
Table 2

Photometric Follow-up Observations of KELT-23Ab

\begin{tabular}{|c|c|c|c|c|c|c|c|c|c|}
\hline Observatory & Location & $\begin{array}{l}\text { Aperture } \\
\text { (m) }\end{array}$ & $\begin{array}{l}\text { Plate Scale } \\
\left(" \mathrm{pix}^{-1}\right)\end{array}$ & $\begin{array}{l}\text { Date } \\
\text { (UT 2018) }\end{array}$ & Filter & $\begin{array}{l}\text { Exposure } \\
\text { Time (s) }\end{array}$ & $\begin{array}{l}\mathrm{rms}^{\mathrm{a}} \\
\left(10^{-3}\right)\end{array}$ & $\begin{array}{l}\text { Detrending } \\
\text { Parameters }\end{array}$ & $\begin{array}{l}\text { Transit } \\
\text { Coverage }\end{array}$ \\
\hline WCO & PA, USA & 0.35 & 0.45 & 26 January & $I$ & 90 & 2.42 & $\begin{array}{l}\text { Airmass, total } \\
\text { counts, Y }\end{array}$ & Full transit \\
\hline PvdK & PA, USA & 0.61 & 0.38 & 26 January & $r^{\prime}$ & 60 & 2.67 & Airmass, FWHM & Full transit \\
\hline DEMONEXT & AZ, USA & 0.50 & 0.90 & 03 February & $g^{\prime}$ & 30 & 4.95 & Airmass, $\mathrm{X}$ & Full transit \\
\hline KeplerCam & AZ, USA & 1.20 & 0.37 & 04 February & $i^{\prime}$ & 15 & 3.58 & Airmass & Mid-transit, egress \\
\hline PvdK & PA, USA & 0.61 & 0.38 & 10 March & $z^{\prime}$ & 60 & 2.86 & Airmass, Y & Full transit \\
\hline ASP & MA, USA & 0.355 & 0.69 & 19 March & $B$ & 30 & 5.80 & $\begin{array}{l}\text { Airmass, total } \\
\text { counts, } \mathrm{X}\end{array}$ & Full transit \\
\hline ASP & MA, USA & 0.355 & 0.69 & 19 March & $R$ & 10 & 4.78 & Airmass, total counts & Full transit \\
\hline WCO & PA, USA & 0.35 & 0.45 & 19 March & $z^{\prime}$ & 120 & 4.60 & Airmass & Full transit \\
\hline SOTES & Suwalki, Poland & 0.10 & 1.65 & 31 May & $V$ & 120 & 4.05 & Airmass, total counts & Full transit \\
\hline
\end{tabular}

Notes.

a The root-mean-square scatter of the best-fit transit model residuals.

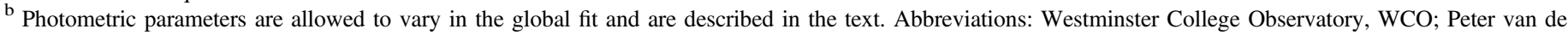

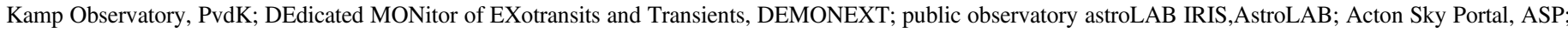
Gabriel Murawski Private Observatory, SOTES; Kutztown University Observatory, KUO.

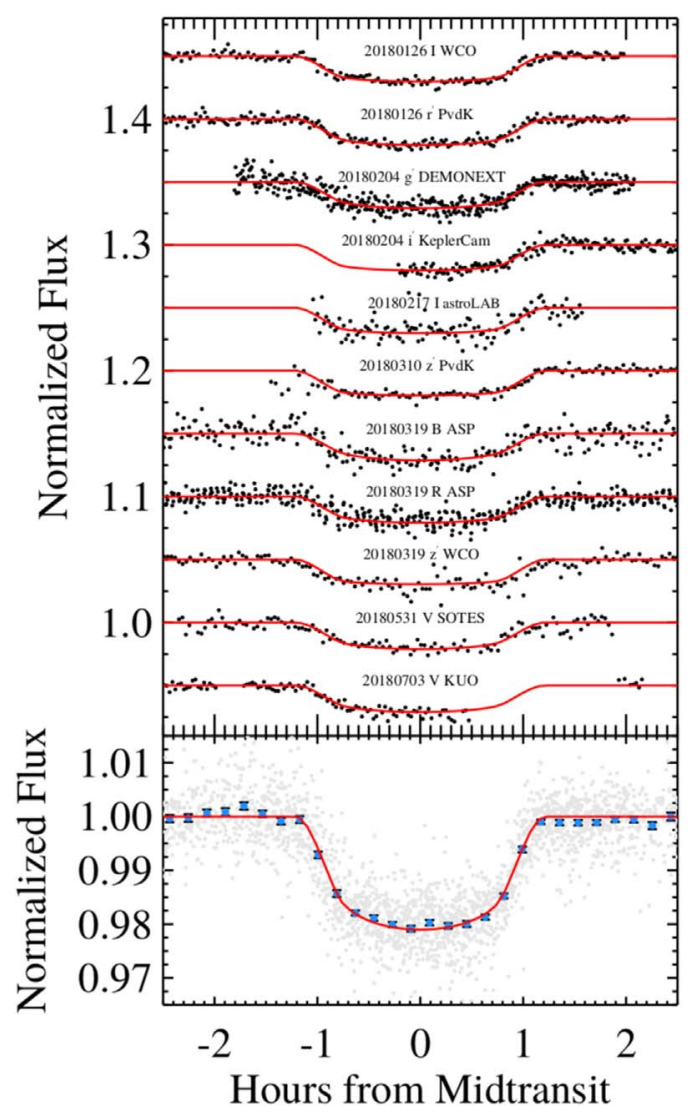

Figure 2. Top: transit light curves of KELT-23Ab from the KELT-North FUN. Observations are offset by an arbitrary amount and are plotted in chronological order with the observatory's acronym (see Table 2) and the date of observation above the light curve. Black dots represent observed relative flux. The red lines represent the fit to each data set. Bottom: all follow-up light curves combined and binned (blue squares). The red line represents the fit to the data. This combined light curve is strictly used to highlight the statistical significance of follow-up observations and is not used for analysis.

\subsection{Spectroscopic Follow-up}

\subsubsection{Tillinghast Reflector Échelle Spectrograph at Fred Lawrence Whipple Observatory}

We obtained 21 spectra from the Tillinghast Reflector Échelle Spectrograph (TRES ${ }^{44}$; Szentgyorgyi \& Fúrész 2007; Fúrész et al. 2008) on the $1.5 \mathrm{~m}$ telescope at the Fred Lawrence Whipple Observatory (FLWO) on Mount Hopkins, Arizona, USA. We observed KELT-23A with TRES between UT 2018 February 18 and UT 2018 June 7. TRES is a 2!" 3 (as projected on the sky) fiber-fed échelle spectrograph with a wavelength dispersion between 3900 and $9100 \AA$ and a resolving power of $R \sim 44,000$. Radial velocities were obtained through the procedure described below. These spectra were also used to calculate bisector spans (BSs) for use in the false-positive analysis in Section 5. The procedure for calculating BSs can be found in detail in Buchhave et al. (2010). The TRES radial velocities and BSs can be seen in Table 3.

Relative radial velocities from TRES are found by first designating the spectrum with the highest signal-to-noise ratio $(\mathrm{S} / \mathrm{N})$ as the reference spectrum. Each individual spectrum is then cross-correlated with the reference. This cross-correlation utilizes the portion of the spectrum between 4300 and $5660 \AA$. To obtain absolute radial velocities from TRES the reference spectrum's Mg b region $(5190 \AA)$ is cross-correlated with the appropriate Kurucz (1992) stellar atmosphere spectrum. From there, absolute radial velocities are adjusted to the International Astronomical Union (IAU) Radial Velocity Standard Star system (Stefanik et al. 1999). This correction is of the order of $-0.61 \mathrm{~km} \mathrm{~s}^{-1}$ and serves to correct for the noninclusion of gravitational redshift in the model spectrum. The absolute radial velocity of the KELT-23A system was found to be $-15.224 \pm 0.10 \mathrm{~km} \mathrm{~s}^{-1}$, where the uncertainty is a result of the residual systematic error in the transformation to the IAU

\footnotetext{
$\overline{44}$ http://tdc-www.harvard.edu/instruments/TRES
} 
Table 3

Absolute Radial Velocities and Bisector Spans for KELT-23Ab

\begin{tabular}{lrrrrl}
\hline \hline BJD & $\begin{array}{r}\text { RV } \\
\left(\mathrm{s}^{-1}\right)\end{array}$ & $\begin{array}{r}\sigma_{\mathrm{RV}} \\
\left(\mathrm{s}^{-1}\right)\end{array}$ & $\begin{array}{r}\text { Bisector } \\
\left(\mathrm{s}^{-1}\right)\end{array}$ & $\begin{array}{r}\sigma_{\text {Bisector }} \\
\left(\mathrm{s}^{-1}\right)\end{array}$ & Source \\
\hline 2458168.018618 & -162.7 & 27.3 & 20.0 & 21.8 & TRES \\
2458184.972170 & 116.6 & 24.9 & 19.4 & 23.1 & TRES \\
2458200.902902 & 75.4 & 19.1 & -20.8 & 14.2 & TRES \\
2458201.932662 & -222.8 & 21.0 & -9.1 & 16.1 & TRES \\
2458210.887269 & -200.7 & 20.1 & 48.1 & 13.9 & TRES \\
2458211.973315 & 127.7 & 20.1 & 26.2 & 11.7 & TRES \\
2458218.822284 & 105.3 & 23.6 & 6.7 & 16.3 & TRES \\
2458227.783676 & 101.9 & 21.1 & -11.1 & 14.9 & TRES \\
2458241.834480 & -56.7 & 20.2 & -22.3 & 14.2 & TRES \\
2458243.807620 & 61.0 & 18.6 & 10.9 & 7.8 & TRES \\
2458244.791342 & -174.6 & 20.1 & -6.9 & 11.0 & TRES \\
2458245.744880 & 89.4 & 23.1 & -44.5 & 25.2 & TRES \\
2458255.919545 & -217.4 & 16.3 & -19.1 & 12.1 & TRES \\
2458263.868286 & 72.6 & 13.4 & -2.3 & 6.5 & TRES \\
2458268.747169 & 16.4 & 14.6 & 4.0 & 12.5 & TRES \\
2458269.736791 & -194.2 & 13.7 & -6.9 & 10.1 & TRES \\
2458271.788423 & -204.9 & 14.9 & 8.7 & 8.9 & TRES \\
2458273.749890 & -200.5 & 13.1 & -7.0 & 14.3 & TRES \\
2458274.733876 & -19.2 & 12.3 & -5.0 & 8.6 & TRES \\
2458275.708487 & -53.3 & 13.9 & -10.2 & 10.9 & TRES \\
2458276.745735 & -113.8 & 13.0 & 21.2 & 17.7 & TRES \\
2458167.067925 & 138.308 & 8.055 & 14.9 & 12.7 & APF \\
2458207.851774 & 108.658 & 7.488 & 21.3 & 12.5 & APF \\
2458210.942618 & -130.985 & 10.568 & 42.4 & 12.2 & APF \\
2458222.808525 & 36.404 & 6.853 & 18.2 & 13.1 & APF \\
2458230.805805 & -79.151 & 6.772 & 11.3 & 12.5 & APF \\
2458233.858489 & -49.625 & 7.187 & 21.0 & 12.6 & APF \\
2458275.762324 & -19.782 & 6.392 & 8.4 & 12.4 & APF \\
2458283.751511 & 54.966 & 7.085 & 23.5 & 12.1 & APF \\
2458285.739298 & -59.795 & 6.269 & 23.4 & 12.0 & APF \\
2458288.721088 & 147.346 & 6.433 & 25.7 & 12.5 & APF \\
2458289.708981 & -144.553 & 6.269 & 18.4 & 12.9 & APF \\
\hline & & & & &
\end{tabular}

system. The TRES radial velocities are plotted in Figure 3 in green.

\subsubsection{Automated Planet Finder at Lick Observatory}

We obtained 11 spectra of KELT-23A between UT 2018 February 17 and UT 2018 June 20 from the Levy spectrograph on the 2.4 meter Automated Planet Finder $\left(\mathrm{APF}^{45}\right.$; Vogt et al. 2014) telescope at Lick Observatory, Mount Hamilton, CA. The APF spectra have a resolution of $R \sim 100,000$ and are obtained through a $1^{\prime \prime} \times 3^{\prime \prime}$ slit. Observations were done through an iodine cell, which imprints a forest of absorption lines onto the stellar spectrum to serve as a wavelength and instrumental broadening reference. The procedure for obtaining radial velocities from APF spectra differs from the procedure for TRES and is described in great detail in Fulton et al. (2015). The method of extracting radial velocities from the APF spectra involves deconvolving the stellar spectrum from the absorption line forest of the iodine cell. The deconvolved stellar spectrum is then used to evaluate the Doppler shift and model the instrumental point-spread function (Butler et al. 1996).

\footnotetext{
${ }^{45}$ https://www.ucolick.org/public/telescopes/apf.html
}
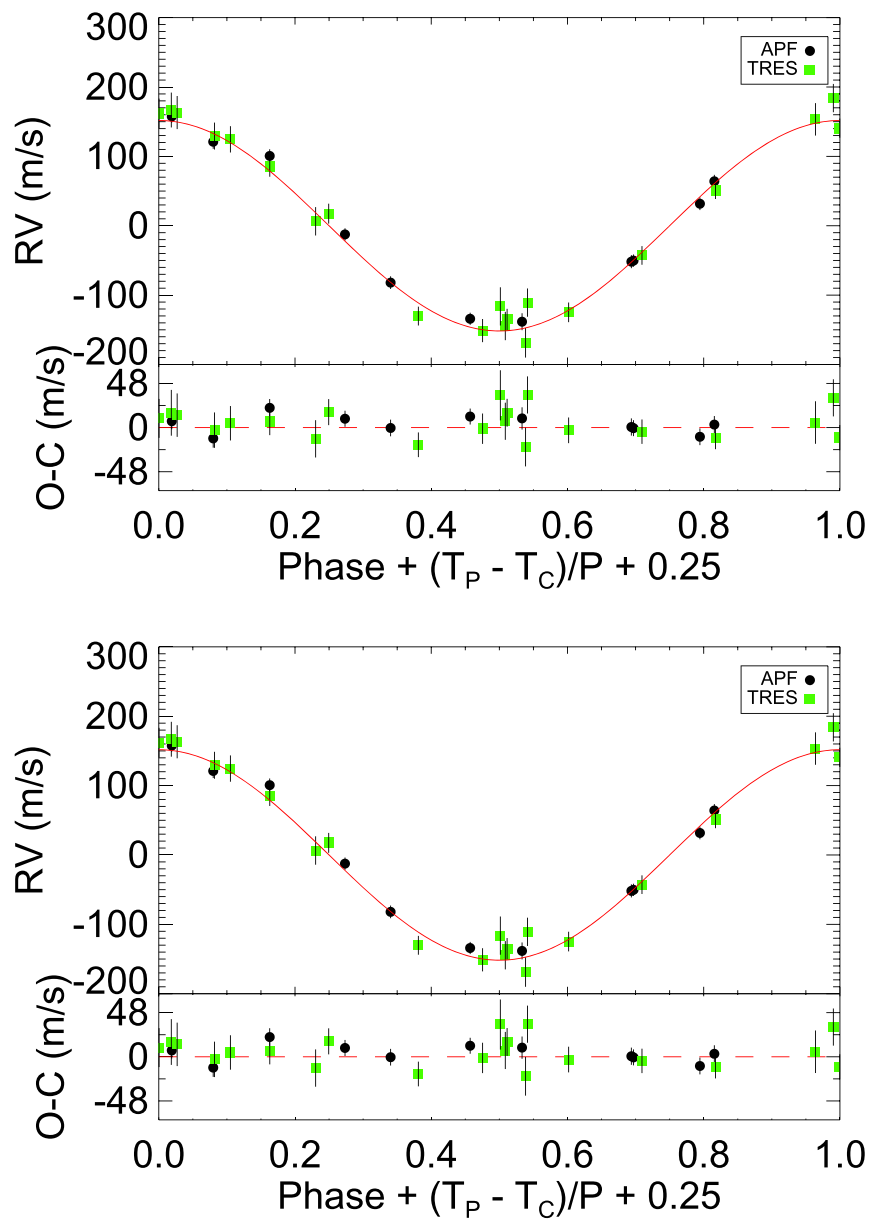

Figure 3. Top: TRES and APF radial velocity measurements. Bottom: TRES and APF radial velocity measurements phase-folded to the period determined by the global fit ( $P=2.25477$ days). Black points represent the APF radial velocities and their uncertainties. Green dots represent the TRES radial velocities and their uncertainties. The red line represents the global fit to the system determined in Section 4.1. The bottom panels show the residuals of the global fit.

\subsection{High-contrast Imaging}

As part of our standard process for validating transiting exoplanets, we observed KELT-23A with infrared highresolution AO imaging at Keck Observatory. The highresolution AO imaging has demonstrated the ability to resolve faint stellar companions, as in the case of the hierarchical triple KELT-21 system (Johnson et al. 2018b). The Keck Observatory observations were made with the NIRC2 instrument on Keck II behind the natural guide star AO system. The observations were made on 2018 June 6 in the standard three-point dither pattern that is used with NIRC2 to avoid the left lower quadrant of the detector, which is typically noisier than the other three quadrants. The dither pattern step size was $3^{\prime \prime}$ and was repeated twice, with each dither offset from the previous dither by $0 . " 5$.

The observations were made in the narrow-band $\mathrm{Br}-\gamma$ filter $\left(\lambda_{o}=2.1686 ; \Delta \lambda=0.0326 \mu \mathrm{m}\right)$ with an integration time of 10 seconds with one co-add per frame for a total of $90 \mathrm{~s}$ on target. The camera was in the narrow-angle mode with a full field of view of $\sim 10^{\prime \prime}$ and a pixel scale of approximately 0."099442 per pixel. The Keck AO observations clearly detected a companion star 4 " 5 to the southwest of the primary star, which is the known star Two Micron All Sky Survey 


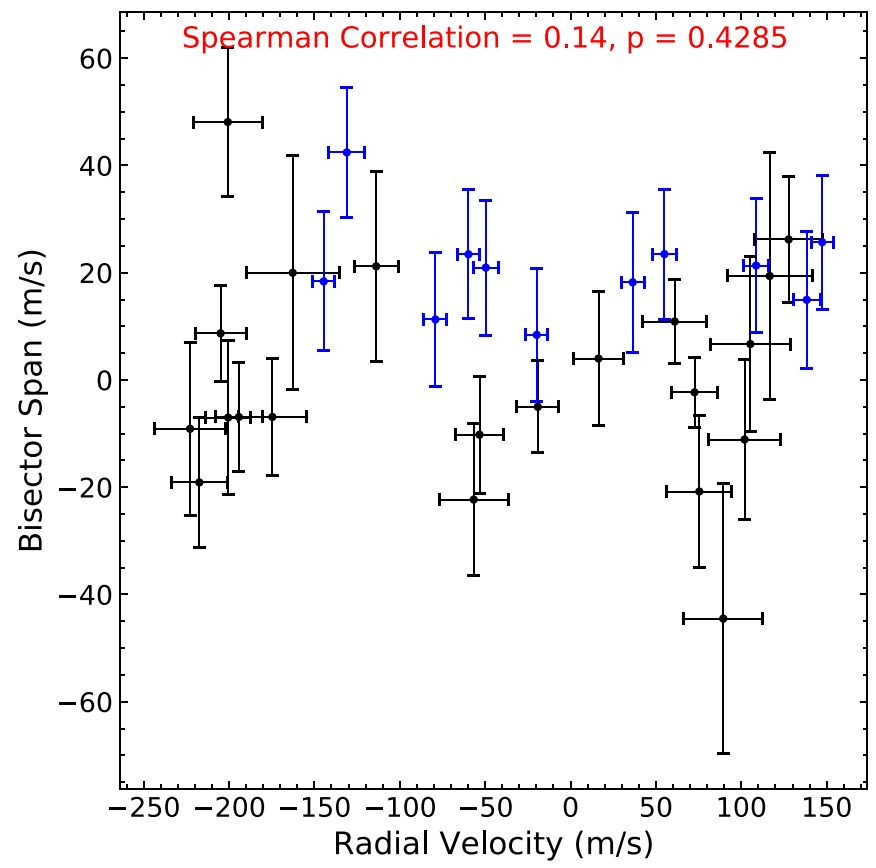

Figure 4. Bisector spans for the TRES (black) and APF (blue) radial velocity spectra plotted against the radial velocity values. There is no statistically significant correlation between these quantities.

(2MASS) J15283577+6621288 (Figure 5) and must be accounted for in the transit depth determinations for observations that do not resolve the stars (Ciardi et al. 2015). No additional stellar companions were detected to within a resolution $\sim 0$ ". 05 FWHM (Figure 6).

The sensitivities of the final combined $\mathrm{AO}$ image were determined by injecting simulated sources azimuthally around the primary target every $45^{\circ}$ at separations of integer multiples of the central source's FWHM (Furlan et al. 2017). The brightness of each injected source was scaled until standard aperture photometry detected it with $5 \sigma$ significance. The resulting brightness of the injected sources relative to the target set the contrast limits at that injection location. The final $5 \sigma$ limit at each separation was determined from the average of all of the determined limits at that separation and the uncertainty on the limit was set by the rms dispersion of the azimuthal slices at a given radial distance. The sensitivity curve is shown in Figure 6 along with an inset image zoomed to the primary target showing no other companion stars.

\section{Host Star Properties}

\subsection{Spectral Analysis}

Spectral analysis allows us to obtain initial estimates of several characteristics of KELT-23A. The TRES spectra were analyzed using the spectral parameter classification (SPC) procedure outlined in Buchhave et al. (2012). SPC crosscorrelates the spectra against a grid of stellar atmospheres made by Kurucz $(1979,1992)$. Allowing SPC to vary all parameters, we obtain a preliminary $T_{\text {eff }}=5915 \pm 50 \mathrm{~K}$, $\log g_{\star}=4.45 \pm 0.10 \quad(\mathrm{cgs}),[\mathrm{Fe} / \mathrm{H}]=-0.09 \pm 0.08$, and $v \sin I_{*}=2.42 \pm 0.502 \mathrm{~km} \mathrm{~s}^{-1}$. The errors reported represent mean errors. The low $v \sin I_{*}$ value indicates that the spectra are not significantly broadened by stellar rotation. The APF spectra were also analyzed and yielded a stellar effective temperature of $5775 \pm 110 \mathrm{~K}$ and $\mathrm{a}[\mathrm{Fe} / \mathrm{H}]$ of $-0.03 \pm 0.06$. The larger

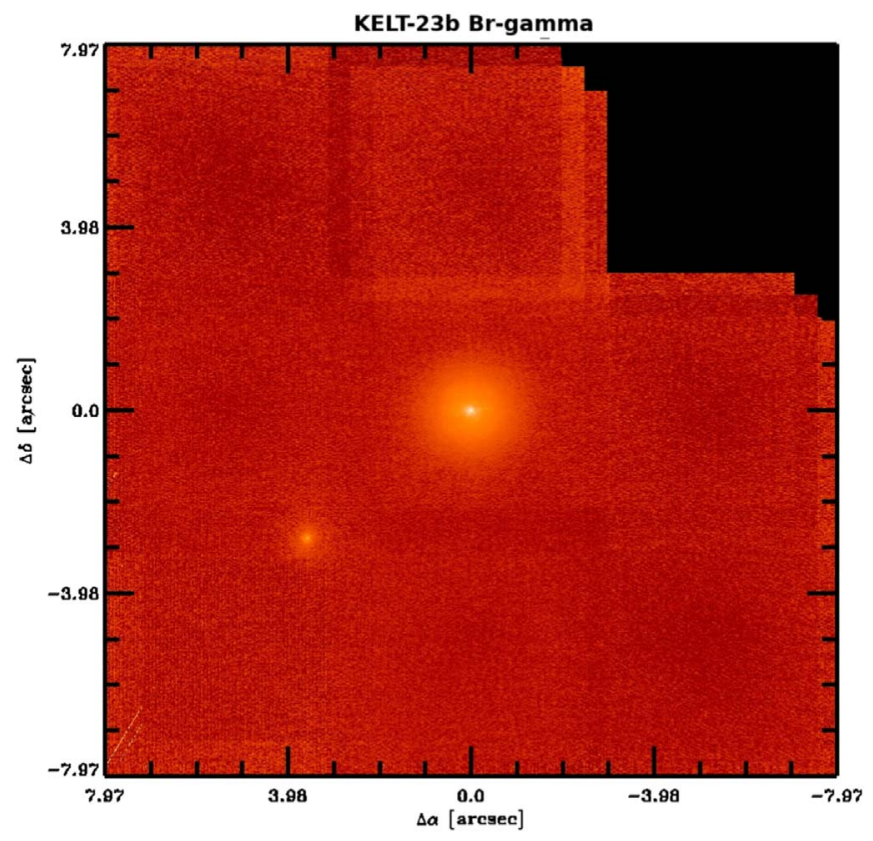

Figure 5. Full field-of-view image of the final combined dither pattern for the Keck OA imaging. The image clearly shows both known 2MASS stars but no other stars within $\sim 10^{\prime \prime}$.

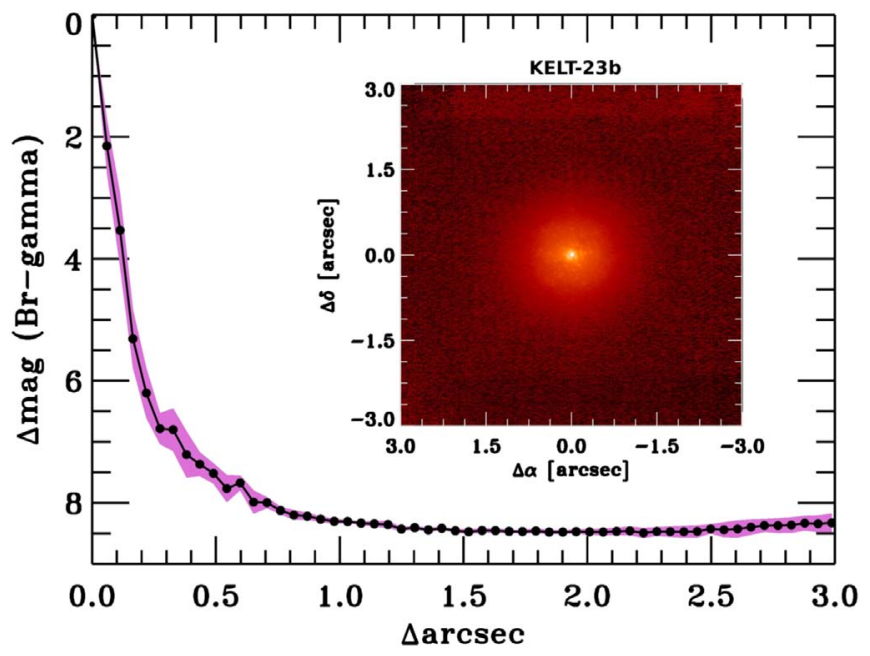

Figure 6. Companion sensitivity for the Keck AO imaging. The black points represent the $5 \sigma$ limits and are separated in steps of 1 FWHM $(\sim 0$." 05); the purple represents the azimuthal dispersion $(1 \sigma)$ of the contrast determinations (see the text). The inset image is of the primary target showing no additional companions to within $3^{\prime \prime}$ of the target.

quantity and $\mathrm{S} / \mathrm{N}$ of the TRES spectra led us to accept the values obtained by the SPC over those obtained on the APF spectra.

\subsection{Spectral Energy Distribution Analysis}

Using KELT-23A's spectral energy distribution (SED) together with the Gaia parallax, we were able to determine an empirical constraint on its radius. We use broadband photometry, shown in Table 1, spanning the wavelength range 0.2-22 $\mu \mathrm{m}$ from the Galaxy Evolution Explorer near-UV to the WISE4 passbands. We excluded the 2MASS $H$ and $K_{S}$ bands because they contained the "U" quality flag in the 2MASS catalog, meaning that the magnitude listed is an upper limit. We 
adopted the $T_{\text {eff }}$ and $[\mathrm{Fe} / \mathrm{H}]$ from the spectrosopic analysis (see Section 3.1), such that the one remaining free parameter of the fit is extinction, $A_{V}$. We use the stellar atmosphere models of Kurucz $(1979,1992)$ to fit the SED, and the bolometric flux $F_{\text {bol }}$ is obtained by numerically integrating the (unreddened) fitted SED model. The existence of a known companion within 4."5 (Section 2.4) indicates that the fluxes in the above bandpasses are blended, so we simultaneously fitted the companion star's SED by enforcing the same distance and extinction (see Section 4.4) but in this case fitting for $T_{\text {eff }}$, and then subtracting the companion star's $F_{\text {bol }}$ in order to obtain the true $F_{\text {bol }}$ for KELT-23A alone.

We obtain a good fit with reduced $\chi^{2}=4.4$, resulting in a best fit of $A_{V}=0.075 \pm 0.015$ and a (companion fluxcorrected) $F_{\text {bol }}$ for KELT-23A of $(2.056 \pm 0.071) \times 10^{-9} \mathrm{erg}$ $\mathrm{s}^{-1} \mathrm{~cm}^{-2}$. With the Gaia DR2 parallax (corrected for the systematic offset from Stassun \& Torres 2018), we obtain a preliminary stellar radius for KELT-23A of $0.959 \pm 0.016 R_{\odot}$.

\subsection{Stellar Models and Age}

We estimate the age of KELT-23A using the stellar parameters determined from the circular Modules for Experiments in Stellar Astrophysics (MESA) Isochrones and Stellar Tracks (MIST; Paxton et al. 2011, 2013, 2015; Choi et al. 2016; Dotter 2016) model fit in Section 4.1 and Table 4. We also determine an evolutionary track for KELT-23A, using a similar process to that in Siverd et al. (2012). In short, we find the intersection of the evolutionary track corresponding to the $M_{\star}$ and $[\mathrm{Fe} / \mathrm{H}]$ with the best fitting isochrone for our $T_{\text {eff }}$ and $\log g_{\star}$, as displayed in Figure 8. We find an age of $6.3_{-3.2}^{+3.5} \mathrm{Gyr}$, where the uncertainty only accounts for observational uncertainty in the parameters used in the fit. This does not account for systematic uncertainties or calibration uncertainties in the MIST model. We find that KELT-23A is a main-sequence G2V-type star in the second half of its lifetime on the main sequence, making KELT-23A a slightly older solar-type star.

The star's metallicity suggests that it could also be slightly subsolar. A more detailed analysis of individual elemental abundances of the host star is encouraged in order to search for deviations from the solar abundance ratios.

\section{Analysis and Results}

\subsection{EXOFASTv2 Global Fit}

In order to gain a more complete understanding of the parameters of the KELT-23A system, we simultaneously fit the ground-based photometric observations from the KELT-FUN (See Figure 2) and the radial velocities (See Figure 3) from TRES using EXOFASTv2 (Eastman et al. 2013; Eastman 2017). Within the global fit, the MIST stellar evolution models are simultaneously fit to constrain the stellar parameters. We place a Gaussian prior on the $[\mathrm{Fe} / \mathrm{H}]$ of $-0.09 \pm 0.08$ and $T_{\text {eff }}$ of $5915 \pm 50 \mathrm{~K}$ from the SPC analysis of the TRES and APF spectra (See Section 3.1). We also place a Gaussian prior on $R_{\star}$ from the value given by the SED analysis (see Section 3.2, Figure 7). We perform two separate fits of KELT-23Ab, one where eccentricity is a free parameter and the other where eccentricity is enforced to be zero. The results of our two fits are shown in Tables 4 and 5 .

\subsection{UVW Space Motion}

We computed the three-dimensional space motion of KELT23A in Galactic coordinates (UVW) to characterize it kinematically in a Galactic context. The systemic velocity was measured to be $-15.224 \pm 0.10 \mathrm{~km} \mathrm{~s}^{-1}$ (Section 2.3), which is within $1 \sigma$ of the value reported by Gaia DR2 $\left(\mathrm{RV}_{\text {Gaia }}=-14.891 \pm 0.379\right.$; Katz et al. 2019), and the proper motion of the system was found to be $\mu_{\alpha}=0.434 \pm 0.039$ mas yr$^{-1}$ and $\mu_{\delta}=-12.217 \pm$ $0.041 \mathrm{mas} \mathrm{yr}^{-1}$ (Table 1). We adopt the distance value derived from the Gaia parallax (Table 1), and the standard of rest values from Coşkunoğlu et al. (2011). We calculate the space motion to be $(U, V, W)=(17.32 \pm 0.03, \quad 0.95 \pm 0.07, \quad-0.90 \pm$ $0.07) \mathrm{km} \mathrm{s}^{-1}$. When compared with the distributions in Bensby et al. (2003), we find a $99.4 \%$ probability that KELT-23A is a member of the thin-disk population in the Galaxy. KELT-23A's near-solar metallicity and relatively low velocities are consistent with the age derived in Section 3.3.

\subsection{Transit Timing Variation Results}

Transit timing variation (TTVs) can be evidence of an unobserved planetary companion gravitationally perturbing the observed planet (Agol et al. 2005; Holman \& Murray 2005). Hot Jupiters seldom show significant TTVs because they rarely have companions close to their mean motion resonances (Steffen et al. 2012). However, there is at least one example of a hot Jupiter that exhibits TTVs due to a nearby companion, specifically WASP-47b, which was discovered by Hellier et al. (2012) and whose TTVs due to a nearby Neptune-mass companion were discovered in K2 data by Becker et al. (2015). Therefore, it is important that we continue to search for hot Jupiters with detectable TTVs, as these rare systems can provide rich and precious information about the emplacement mechanisms of hot Jupiters.

To analyze any possible TTVs we first converted each observatory's photometric time stamps to $\mathrm{BJD}_{\mathrm{TDB}}$ (Eastman et al. 2010) before the global fit in Section 4.1. The accuracy of the time stamps is assured by the synchronization to a standard clock by each observatory during observations. This synchronization assures that any uncertainty in $T_{0}$ is a result of the global fit. We then plotted the $\mathrm{O}-\mathrm{C}$ residuals between the observed transit times and the calculated transit times using the ephemeris determined in Section 4.1 (Table 4). The O-C residuals are in Table 6 and plotted in Figure 9. Similar to the KELT-14 TTV analysis (Rodriguez et al. 2016), it would appear that there are significant TTVs. However, observatorydependent factors such as seeing conditions and astrophysical red noise (Carter \& Winn 2009) can induce discrepancies in the observed transit mid-times. Due to these factors, we do not claim that the observed TTVs are astrophysical in nature and conclude that they are likely spurious and a result of systematics. Continued monitoring, especially at the precision that will be reached by TESS, will unveil any true TTVs.

\subsection{Evidence for a Wide Binary Star System}

As with some other recently discovered hot-Jupiter hosts, such as KELT-18 (McLeod et al. 2017), there is evidence that KELT-23A is a member of a wide binary star system. The nearby star described in Section 2.4 is most likely gravitationally bound to KELT-23A. The agreement of the parallaxes of both components within their sub-one-percent uncertainties, 
Table 4

Median Values and 68\% Confidence Intervals for the Physical and Orbital Parameters of the KELT-23A System

\begin{tabular}{|c|c|c|c|}
\hline Parameter & Units & $\begin{array}{c}\text { Value } \\
\text { (MIST eccentric) }\end{array}$ & $\begin{array}{c}\text { Adopted Value } \\
\text { (MIST circular; } e=0 \text { fixed) }\end{array}$ \\
\hline \multicolumn{4}{|l|}{ Stellar Parameters: } \\
\hline$M_{*}$ & Mass $\left(M_{\odot}\right)$ & $0.942_{-0.055}^{+0.062}$ & $0.944_{-0.054}^{+0.060}$ \\
\hline$R_{*}$ & Radius $\left(R_{\odot}\right)$ & $0.996 \pm 0.015$ & $0.996 \pm 0.015$ \\
\hline$L_{*}$ & Luminosity $\left(L_{\odot}\right)$ & $1.082_{-0.049}^{+0.051}$ & $1.081_{-0.048}^{+0.051}$ \\
\hline$\rho_{*}$ & Density (cgs) & $1.345_{-0.087}^{+0.098}$ & $1.349_{-0.083}^{+0.090}$ \\
\hline $\log g$ & Surface gravity (cgs) & $4.416_{-0.027}^{+0.028}$ & $4.417_{-0.025}^{+0.026}$ \\
\hline$T_{\text {eff }}$ & Effective temperature (K) & $5899 \pm 49$ & $5899 \pm 49$ \\
\hline$[\mathrm{Fe} / \mathrm{H}]$ & Metallicity (dex) & $-0.106_{-0.077}^{+0.078}$ & $-0.105_{-0.077}^{+0.078}$ \\
\hline$[\mathrm{Fe} / \mathrm{H}]_{0}$ & Initial metallicity & $-0.072_{-0.071}^{+0.070}$ & $-0.071 \pm 0.071$ \\
\hline Age & Age (Gyr) & $6.5_{-3.3}^{+3.7}$ & $6.4_{-3.2}^{+3.5}$ \\
\hline EEP & Equal evolutionary point & $374_{-32}^{+27}$ & $374_{-30}^{+27}$ \\
\hline$\dot{\gamma}$ & Radial velocity slope $\left(\mathrm{m} \mathrm{s}^{-1}\right.$ day $\left.^{-1}\right)$ & $-0.196_{-0.090}^{+0.079}$ & $-0.276_{-0.11}^{+0.098}$ \\
\hline \multicolumn{2}{|l|}{ Planetary Parameters: } & $\mathrm{b}$ & $\mathrm{b}$ \\
\hline$P$ & Period (days) & $2.255249 \pm 0.000012$ & $2.255251_{-0.000012}^{+0.000011}$ \\
\hline$R_{P}$ & Radius $\left(R_{\mathrm{J}}\right)$ & $1.323 \pm 0.025$ & $1.323 \pm 0.025$ \\
\hline$T_{C}$ & Time of conjunction $\left(\mathrm{BJD}_{\mathrm{TDB}}\right)$ & $2458007.3184_{-0.0029}^{+0.0027}$ & $2458007.3194_{-0.0028}^{+0.0027}$ \\
\hline$T_{0}$ & Optimal conjunction time $\left(\mathrm{BJD}_{\mathrm{TDB}}\right)$ & $2458140.3781_{-0.0030}^{+0.0028}$ & $2458140.3792_{-0.0029}^{+0.0027}$ \\
\hline$a$ & Semimajor axis (au) & $0.03300_{-0.00065}^{+0.00071}$ & $0.03302_{-0.00064}^{+0.00068}$ \\
\hline$i$ & Inclination (Degrees) & $85.37_{-0.30}^{+0.31}$ & $85.37_{-0.30}^{+0.31}$ \\
\hline$e$ & Eccentricity & $0.036_{-0.013}^{+0.014}$ & $\ldots$ \\
\hline$\omega_{*}$ & Argument of periastron (Degrees) & $3_{-40}^{+36}$ & $\ldots$ \\
\hline$T_{e q}$ & Equilibrium temperature $(\mathrm{K})$ & $1562 \pm 21$ & $1561 \pm 20$ \\
\hline$M_{P}$ & Mass $\left(M_{\mathrm{J}}\right)$ & $0.936_{-0.043}^{+0.047}$ & $0.938_{-0.044}^{+0.048}$ \\
\hline K & RV semi-amplitude $\left(\mathrm{m} \mathrm{s}^{-1}\right)$ & $150.5_{-3.7}^{+3.8}$ & $150.6_{-4.3}^{+4.4}$ \\
\hline $\log K$ & Log of RV semi-amplitude & $2.178 \pm 0.011$ & $2.178_{-0.013}^{+0.012}$ \\
\hline$R_{P} / R_{*}$ & Radius of planet in stellar radii & $0.1365 \pm 0.0011$ & $0.1365 \pm 0.0010$ \\
\hline$a / R_{*}$ & Semimajor axis in stellar radii & $7.13_{-0.16}^{+0.17}$ & $7.13_{-0.15}^{+0.16}$ \\
\hline$\delta$ & Transit depth (fraction) & $0.01863_{-0.00030}^{+0.00031}$ & $0.01864 \pm 0.00028$ \\
\hline Depth & Flux decrement at mid-transit & $0.01863_{-0.00030}^{+0.00031}$ & $0.01864 \pm 0.00028$ \\
\hline$\tau$ & Ingress/egress transit duration (days) & $0.01706_{-0.00097}^{+0.0010}$ & $0.01710_{-0.00085}^{+0.00089}$ \\
\hline$T_{14}$ & Total transit duration (days) & $0.0992 \pm 0.0010$ & $0.09921 \pm 0.00092$ \\
\hline$T_{\text {FWHM }}$ & FWHM transit duration (days) & $0.08211_{-0.00061}^{+0.00060}$ & $0.08208_{-0.00060}^{+0.00061}$ \\
\hline$b$ & Transit impact parameter & $0.575_{-0.031}^{+0.028}$ & $0.576_{-0.027}^{+0.024}$ \\
\hline$b_{S}$ & Eclipse impact parameter & $0.575_{-0.028}^{+0.027}$ & $\ldots$ \\
\hline$\tau_{S}$ & Ingress/egress eclipse duration (days) & $0.0171_{-0.0010}^{+0.0011}$ & $\ldots$ \\
\hline$T_{S, 14}$ & Total eclipse duration (days) & $0.0994_{-0.0025}^{+0.0002}$ & $\ldots$ \\
\hline$T_{S, \text { FWHM }}$ & FWHM eclipse duration (days) & $0.0822_{-0.0017}^{+0.0018}$ & $\ldots$ \\
\hline$\delta_{S, 3.6 \mu m}$ & Blackbody eclipse depth at $3.6 \mu \mathrm{m}$ (ppm) & $1484 \pm 63$ & $1482_{-62}^{+64}$ \\
\hline$\delta_{S, 4.5 \mu m}$ & Blackbody eclipse depth at $4.5 \mu \mathrm{m}$ (ppm) & $1981 \pm 74$ & $1980_{-73}^{+76}$ \\
\hline$\rho_{P}$ & Density (cgs) & $0.502_{-0.035}^{+0.038}$ & $0.503_{-0.036}^{+0.039}$ \\
\hline $\log g_{P}$ & Surface gravity & $3.123_{-0.026}^{+0.027}$ & $3.124 \pm 0.027$ \\
\hline$\Theta$ & Safronov number & $0.0495_{-0.0015}^{+0.0016}$ & $0.0496 \pm 0.0017$ \\
\hline$\langle F\rangle$ & Incident flux $\left(10^{9} \mathrm{erg} \mathrm{s}^{-1} \mathrm{~cm}^{-2}\right)$ & $1.349_{-0.070}^{+0.073}$ & $1.349_{-0.067}^{+0.070}$ \\
\hline$T_{P}$ & Time of periastron $\left(\mathrm{BJD}_{\mathrm{TDB}}\right)$ & $2458006.80_{-0.25}^{+0.23}$ & $2458007.3194_{-0.0028}^{+0.0027}$ \\
\hline$T_{S}$ & Time of eclipse $\left(\mathrm{BJD}_{\mathrm{TDB}}\right)$ & $2458006.234_{-0.017}^{+0.015}$ & $2458008.4470_{-0.0028}^{+0.0027}$ \\
\hline$T_{A}$ & Time of ascending node $\left(\mathrm{BJD}_{\mathrm{TDB}}\right)$ & $2458006.776 \pm 0.018$ & $2458006.7556_{-0.0028}^{+0.0027}$ \\
\hline$T_{D}$ & Time of descending node $\left(\mathrm{BJD}_{\mathrm{TDB}}\right)$ & $2458007.901_{-0.017}^{+0.019}$ & $2458007.8832_{-0.0028}^{+0.0027}$ \\
\hline$e \cos \omega_{*}$ & & $0.030_{-0.012}^{+0.011}$ & $\ldots$ \\
\hline$e \sin \omega_{*}$ & & $0.001 \pm 0.021$ & $\ldots$ \\
\hline$M_{P} \sin i$ & Minimum mass $\left(M_{\mathrm{J}}\right)$ & $0.933_{-0.043}^{+0.047}$ & $0.935_{-0.044}^{+0.048}$ \\
\hline$M_{P} / M_{*}$ & Mass ratio & $0.000948 \pm 0.000030$ & $0.000949 \pm 0.000033$ \\
\hline$d / R_{*}$ & Separation at mid-transit & $7.11_{-0.25}^{+0.27}$ & $7.13_{-0.15}^{+0.16}$ \\
\hline$P_{T}$ & A priori nongrazing transit prob. & $0.1215_{-0.0044}^{+0.0045}$ & $0.1210 \pm 0.0025$ \\
\hline$P_{T, G}$ & A priori transit prob. & $0.1599_{-0.0058}^{+0.0059}$ & $0.1593 \pm 0.0035$ \\
\hline$P_{S}$ & A priori nongrazing eclipse prob. & $0.1211 \pm 0.0029$ & $\ldots$ \\
\hline$P_{S, G}$ & A priori eclipse prob. & $0.1594_{-0.0041}^{+0.0042}$ & $\ldots$ \\
\hline
\end{tabular}




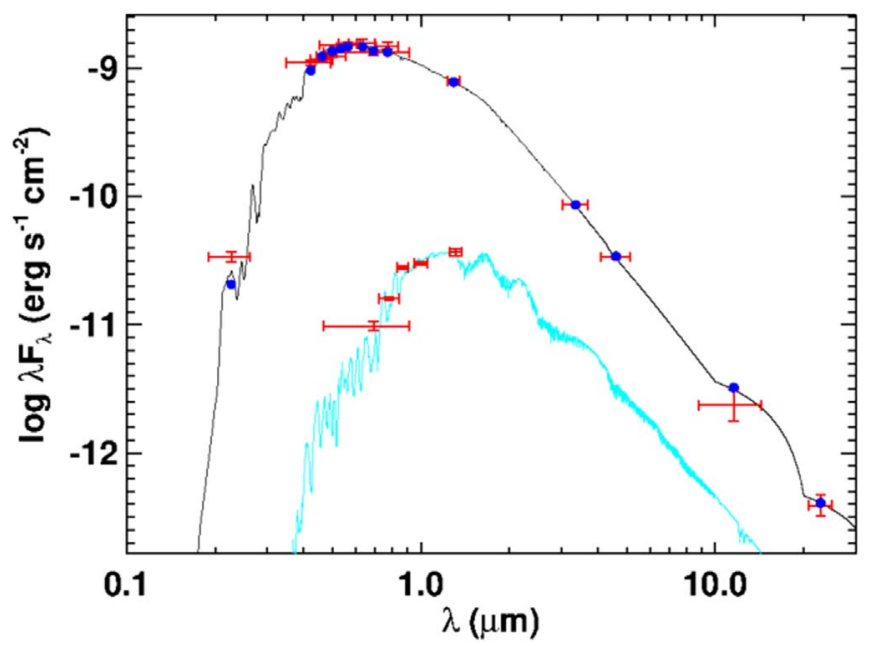

Figure 7. SED fits for KELT-23A (black line) and the fainter companion within 4!. 5 (Section 2.4; light blue line). Red dots on KELT-23A's SED represent observed values for each bandpass in Table 1. Horizontal errors represent the width of each bandpass and vertical errors represent the $1 \sigma$ uncertainty of the fit. The blue dots represent the points of the model fit. Red dots on the SED of the companion result from our AO analysis, Gaia measurements, and Pan-STARRS measurements.

and the consistency of their relative proper motion with their expected orbital motion, lead us to conclude that the nearby star is bound to KELT-23A.

The nearby star's Gaia DR2 parallax (also corrected for the systematic offset from Stassun \& Torres 2018) is $7.977 \pm 0.052$ mas and places the star at a distance of $125.36 \pm 0.82 \mathrm{pc}$, equal to KELT-23A's Gaia-derived distance to within the small uncertainty. At that distance, with an apparent angular separation of 4.15 , the projected (minimum) distance between the two stellar components would be 570 au.

The nearby star's Gaia DR2 proper motions of $\mu_{\alpha}=$ $1.567 \pm 0.092$ mas yr$^{-1}$ and $\mu_{\delta}=-11.902 \pm 0.107$ mas yr$^{-1}$ are close to KELT-23A's (Table 1) and give the two stars a relative proper motion between them of just $1.2{\text { mas } \mathrm{yr}^{-1}}^{-1}$. This corresponds to a relative tangential velocity of $0.72 \mathrm{~km} \mathrm{~s}^{-1}$, which could be produced by orbital motion. For illustration, a $0.25 M_{\odot}$ companion at 570 au from KELT-23A would be in a $12,000 \mathrm{yr}$ orbit. KELT-23A would have a (circular) orbital speed of $0.28 \mathrm{~km} \mathrm{~s}^{-1}$ and the companion's orbital speed would be $1.12 \mathrm{~km} \mathrm{~s}^{-1}$. A $0.5 M_{\odot}$ companion would orbit with a period of $11,000 \mathrm{yr}$ giving KELT-23A and its companion (circular) orbital speeds of $0.52 \mathrm{~km} \mathrm{~s}^{-1}$ and $1.0 \mathrm{~km} \mathrm{~s}^{-1}$, respectively. The SED analysis (Section 3.2) produces relative fluxes for the companion (Table 7) that are consistent with a $\sim 0.3 M_{\odot}$ star.

The presence of KELT-23A's distant companion adds it to a family of systems that are informing the developing theories about the formation of hot Jupiters. One way to drive a gas giant planet inward toward its host star is through eccentric orbital interactions with a distant companion star via KozaiLidov oscillations (Fabrycky \& Tremaine 2007). The planet's orbit would later circularize due to tidal friction as the planet approaches the host star.

\section{False-positive Analysis}

KELT-23Ab underwent the same rigorous vetting process used for all KELT planet candidates. We first obtain KELTFUN photometry of the candidate (Section 2.2). Follow-up observers have instruments capable of observing the target at faster cadences with greater photometric accuracy and greater angular resolution than KELT. In addition, follow-up photometric observations ideally span a large wavelength range in order to check for wavelength dependent transit depths. Our observations span the $B-z^{\prime}$ range and show no wavelength dependent anomalies. All of our observed light curves are well fit with our global planetary model. The model consists of an opaque circular planet of constant achromatic radius on a Keplerian orbit. (Section 4.1).

An indication that our follow-up TRES spectra are contaminated with a close stellar partner would be double absorption lines. Our 11 TRES spectra and 15 APF spectra show no double absorption lines. We find no evidence for a stellar partner blended in the spectra, signifying that the periodic radial velocity signal arises from KELT-23A.

To rule out any other sources of periodic radial velocity anomalies we analyzed the BSs of each spectrum (Buchhave et al. 2010), found in Table 3 and shown in Figure 4. Between the radial velocities and the BSs, we calculated a Spearman correlation of 0.14 and a $p$ value of 0.4285 , indicating that there is no correlation between the BSs and the radial velocities. We conclude that the observed periodic radial velocity changes are due to the reflex motion of the star caused by the transiting companion.

As a final check, the AO images were analyzed for a companion within $5^{\prime \prime}$ of KELT-23A. There exists a close stellar companion within $4 . .5$ of KELT-23A, which was previously known. This companion was not resolved by the photometry and measures were taken to deblend the fluxes by examining the flux ratios between KELT-23A and the stellar companion in the Johnson-Cousins and Sloan Digital Sky Survey (SDSS) filters. Through the previously mentioned checks, we conclude that the photometric anomalies and spectroscopic radial velocity signal are best described by a planet orbiting KELT-23A.

\section{Discussion}

\subsection{Tidal Evolution and Irradiation History}

We simulate the past and future evolution of KELT-23Ab's orbit using the parameters derived in Section 4.1 as boundary conditions. We use the POET code for this simulation (Penev et al. 2014) under several assumptions: a circular orbit, no perturbing companion planets, and a constant phase lag (constant tidal quality factor). The simulation analyzes the change in the semimajor axis over time due to the tidal forces exchanged by the star and planet. The simulation also analyzes the change in incident flux received by the planet over time. This change in incident flux is a result of both the changing semimajor axis and host-star luminosity as it evolves. Tidal force strengths are parameterized through the tidal dissipation parameter, $Q_{*}^{\prime}$. This parameter is defined as the quotient of the tidal quality factor $\left(Q_{*}\right)$ and the Love number $\left(\mathrm{k}_{2}\right)$. We test values of $Q_{*}^{\prime}$ ranging from $10^{5}$ to $10^{8}$ stepping up only the order of magnitude. This large range in tidal dissipation factor allows us to probe a large range of timescales for energy dissipation. These timescales correspond to the large range of proposed mechanisms for tidal dissipation.

If tides have been the primary cause of changing orbital properties, then KELT-23Ab has been above the inflation irradiation threshold, as defined in Demory \& Seager (2011) $\left(2 \times 10^{8} \mathrm{erg} \mathrm{s}^{-1} \mathrm{~cm}^{-2}\right)$, for its entire history, regardless of the 
Table 5

Median Values and 68\% Confidence Intervals for the Additional Parameters of KELT-23A from EXOFASTv2

\begin{tabular}{|c|c|c|c|c|c|c|}
\hline \multicolumn{7}{|c|}{ Wavelength Parameters: } \\
\hline & $\begin{array}{c}\text { Linear Limb-darkening } \\
u_{1}\end{array}$ & $\begin{array}{l}\text { Quadratic Limb-darkening } \\
u_{2}\end{array}$ & $\begin{array}{c}\text { Dilution } \\
A_{D}\end{array}$ & & & \\
\hline$B$ & $0.612 \pm 0.050$ & $0.202 \pm 0.050$ & $0.0013336 \pm 0.0000067$ & & & \\
\hline I & $0.268 \pm 0.034$ & $0.292 \pm 0.034$ & $0.002565 \pm 0.000013$ & & & \\
\hline$R$ & $0.342 \pm 0.047$ & $0.289 \pm 0.048$ & $0.003504 \pm 0.000018$ & & & \\
\hline$g^{\prime}$ & $0.512_{-0.046}^{+0.047}$ & $0.227 \pm 0.047$ & $0.0014614 \pm 0.0000073$ & & & \\
\hline$i^{\prime}$ & $0.315_{-0.049}^{+0.050}$ & $0.312 \pm 0.049$ & $0.009836 \pm 0.000049$ & & & \\
\hline$r^{\prime}$ & $0.374 \pm 0.045$ & $0.300 \pm 0.047$ & $0.003357 \pm 0.000017$ & & & \\
\hline$z^{\prime}$ & $0.200 \pm 0.034$ & $0.258 \pm 0.034$ & $0.02120 \pm 0.00011$ & & & \\
\hline$V$ & $0.448 \pm 0.036$ & $0.300 \pm 0.035$ & $0.002566 \pm 0.000013$ & & & \\
\hline \multicolumn{7}{|c|}{ Telescope Parameters: } \\
\hline & & \multicolumn{2}{|c|}{ Mist Eccentric } & \multicolumn{2}{|c|}{ Mist Circular } & \\
\hline & & $\overline{\mathrm{APF}}$ & TRES & APF & TRES & \\
\hline$\gamma_{\mathrm{rel}}$ & Relative radial velocity offset $\left(\mathrm{m} \mathrm{s}^{-1}\right)$ & $7.2_{-3.8}^{+4.0}$ & $-59.7_{-4.5}^{+4.8}$ & $7.6_{-5.2}^{+5.5}$ & $-56.1_{-4.8}^{+4.9}$ & \\
\hline$\sigma_{J}$ & $\mathrm{RV}$ jitter $\left(\mathrm{m} \mathrm{s}^{-1}\right)$ & $9.1_{-4.3}^{+5.5}$ & $5.4_{-5.4}^{+7.4}$ & $14.4_{-4.3}^{+6.3}$ & $8.4_{-8.4}^{+6.5}$ & \\
\hline$\sigma_{J}^{2}$ & $\mathrm{RV}$ jitter variance & $82_{-60}^{+130}$ & $28_{-78}^{+130}$ & $210_{-110}^{+220}$ & $70_{-89}^{+150}$ & \\
\hline \multicolumn{7}{|c|}{ Transit Parameters (MIST Circular Fit): } \\
\hline Observation & $\begin{array}{l}\text { Added variance } \\
\qquad \sigma^{2}\end{array}$ & $\begin{array}{l}\text { TTV (days) } \\
\text { (days) }\end{array}$ & $\begin{array}{c}\text { Baseline flux } \\
F_{0} \\
\end{array}$ & $\begin{array}{c}\text { Detrending coeff. } \\
C_{0} \\
\end{array}$ & $\begin{array}{c}\text { Detrending coeff. } \\
\qquad C_{1} \\
\end{array}$ & $\begin{array}{l}\text { Detrending coeff. } \\
\qquad C_{2} \\
\end{array}$ \\
\hline WCO UT 2018-01-26 (I) & $-0.00000126_{-0.00000062}^{+0.0000071}$ & $0.0088_{-0.0028}^{+0.0029}$ & $0.99953 \pm 0.00022$ & $-0.00120_{-0.00061}^{+0.00062}$ & $-0.00034_{-0.00046}^{+0.00047}$ & $-0.00117_{-0.00063}^{+0.00062}$ \\
\hline PvdK UT 2018-01-26 $\left(r^{\prime}\right)$ & $0.00000673_{-0.00000068}^{+0.00000077}$ & $0.0076_{-0.0028}^{+0.0029}$ & $1.00025 \pm 0.00021$ & $-0.00067 \pm 0.00060$ & $-0.00089 \pm 0.00068$ & $-0.00061 \pm 0.00061$ \\
\hline DEMONEXT UT 2018-02-03 $\left(\mathrm{g}^{\prime}\right)$ & $0.0000231_{-0.0000018}^{+0.0000019}$ & $0.0073_{-0.0028}^{+0.0029}$ & $1.00001 \pm 0.00028$ & $-0.00017 \pm 0.00064$ & $0.00079_{-0.00064}^{+0.00063}$ & $\ldots$ \\
\hline KeplerCam UT 2018-02-04 (í) & $0.0000132_{-0.0000013}^{+0.000015}$ & $0.0062_{-0.0028}^{+0.0029}$ & $0.99974 \pm 0.00031$ & $0.00022 \pm 0.00049$ & $\ldots$ & $\cdots$ \\
\hline astroLAB UT 2018-02-17 (I) & $0.0000002_{-0.0000051}^{+0.0000061}$ & $0.0059_{-0.0028}^{+0.0029}$ & $1.00163_{-0.00063}^{+0.00064}$ & $-0.0014 \pm 0.0016$ & $-0.0012 \pm 0.0016$ & $\cdots$ \\
\hline PvdK UT 2018-03-10 (z') & $0.00000648_{-0.00000099}^{+0.0000011}$ & $0.0062_{-0.0029}^{+0.0030}$ & $0.99924 \pm 0.00025$ & $0.00081 \pm 0.00072$ & $0.00061 \pm 0.00073$ & $\ldots$ \\
\hline ASP UT 2018-03-19 (B) & $0.0000218_{-0.0000039}^{+0.0000043}$ & $0.0099_{-0.0030}^{+0.0032}$ & $0.99946_{-0.00052}^{+0.00051}$ & $-0.0011 \pm 0.0014$ & $-0.0025 \pm 0.0016$ & $-0.0018 \pm 0.0014$ \\
\hline ASP UT 2018-03-19 $(R)$ & $0.0000126_{-0.0000017}^{+0.000019}$ & $0.0106_{-0.0029}^{+0.0030}$ & $1.00072 \pm 0.00027$ & $-0.00143_{-0.00072}^{+0.00073}$ & $-0.00108 \pm 0.00073$ & $\ldots$ \\
\hline WCO UT 2018-03-19 (z') & $0.0000008_{-0.0000016}^{+0.0000019}$ & $0.0138_{-0.0032}^{+0.0034}$ & $1.00005_{-0.00062}^{+0.00061}$ & $0.00071_{-0.0010}^{+0.00099}$ & $\ldots$ & $\cdots$ \\
\hline SOTES UT 2018-05-31 ( V ) & $0.0000055_{-0.0000020}^{+0.0000025}$ & $0.0131_{-0.0033}^{+0.0034}$ & $1.00059 \pm 0.00041$ & $-0.00183_{-0.00096}^{+0.00095}$ & $0.00035 \pm 0.00097$ & $\cdots$ \\
\hline KUO UT 2018-07-03 (V) & $0.00000294_{-0.00000074}^{+0.0000084}$ & $0.0121_{-0.0032}^{+0.0034}$ & $0.99865 \pm 0.00032$ & $-0.00298_{-0.00095}^{+0.00094}$ & $-0.00147_{-0.00097}^{+0.00096}$ & $\cdots$ \\
\hline
\end{tabular}




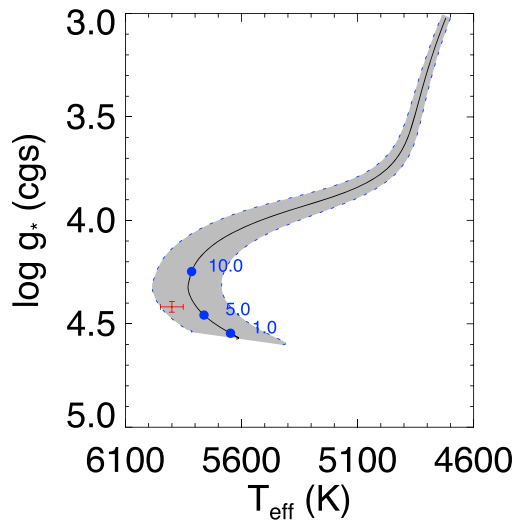

Figure 8. Age determination for KELT-23A. Plotted are KELT-23A with $1 \sigma$ uncertainties (red) and the best fitting MIST track (gray) with the shaded region representing $1 \sigma$ uncertainties on the host star's mass from our global fit. The $M_{\star}$ and $[\mathrm{Fe} / \mathrm{H}]_{0}$ of the host star are determined in Section 4.1 and are used in the Hertzsprung-Russell (HR) diagram analysis (Section 3.3). Blue dots represent the position along the track at 1.0, 5.0, and 10.0 Gyr.

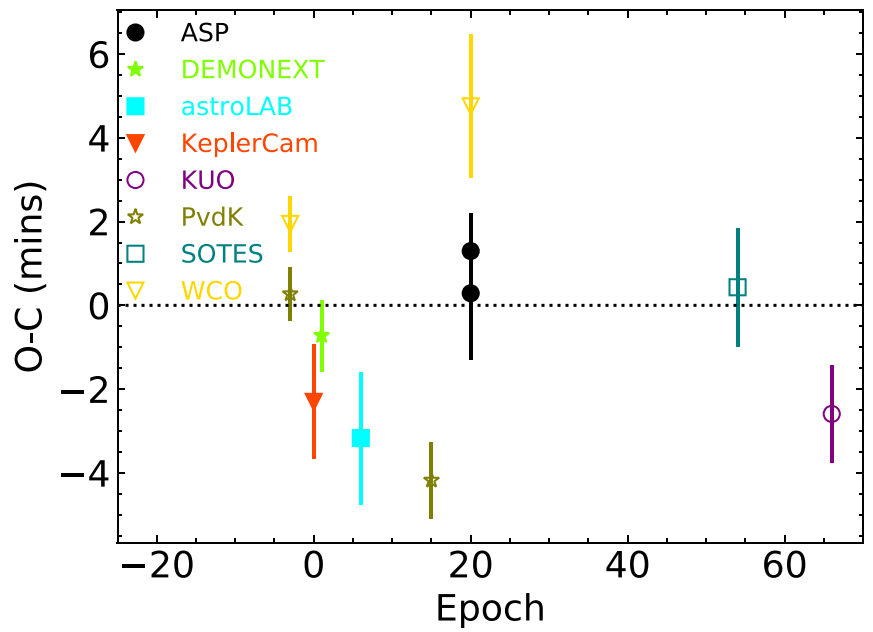

Figure 9. Transit time residuals for KELT-23Ab using the global fit ephemeris determined in Section 4.1. The data are listed in Table 6.

$Q_{*}^{\prime}$ chosen. Currently, KELT-23Ab receives an incident flux about six times larger than the Demory \& Seagar inflation threshold (Figure 10, upper panel). KELT-23Ab's large radius, $R_{\mathrm{P}}=1.322 \pm 0.025 R_{\mathrm{J}}$, is almost certainly due to its long history of high stellar irradiation.

The future of KELT-23Ab is highly dependent on the value of $Q_{*}^{\prime}$. A large value $\left(10^{8}\right)$ will keep the planet in orbit for several gigayears. A small value $\left(10^{5}\right)$ will quickly cause the planet to spiral into its host star, as seen in the bottom panel of Figure 10.

If, in addition to the orbital properties, the spin of the star is known, then it is possible to constrain $Q_{*}^{\prime}$, following the procedure of Penev et al. (2018). Briefly, the procedure relies on comparing the observed spin of the star to the one predicted by gyrochronology and assuming that any excess spin is due to tidal dissipation transferring orbital angular momentum to the star. Not having a measurement of the stellar spin, we can instead simply evaluate the frequency-dependent tidal dissipation found in Penev et al. (2018) at the tidal period of this system, resulting in an expected value of $\log \left(Q_{*}^{\prime}\right)=5.8$. If this truly is the case, then KELT-23Ab is expected to spiral into its host within the next gigayear or so.
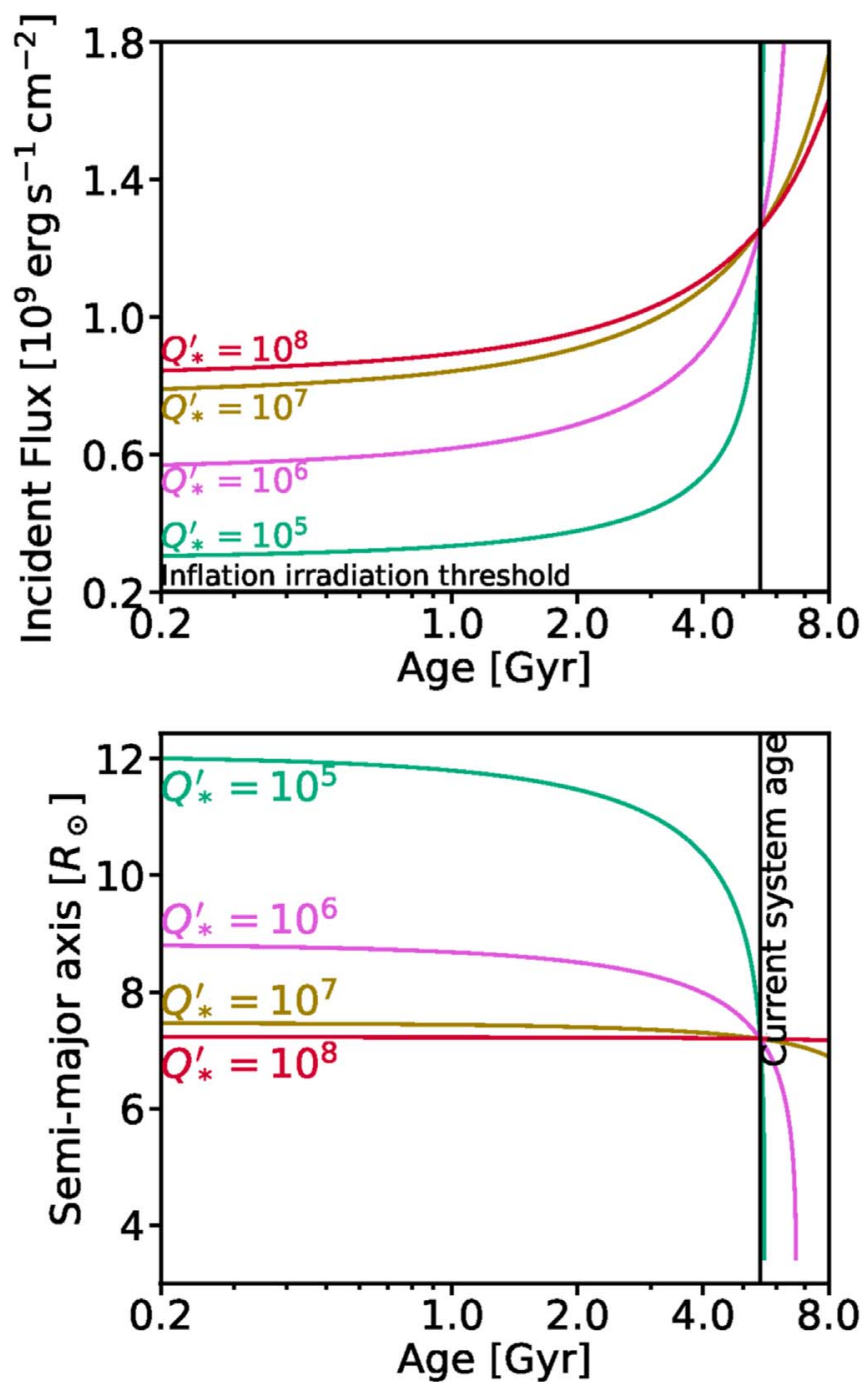

Figure 10. Top: irradiation history of KELT-23Ab modeled for a range of stellar tidal quality factors, $Q_{*}^{\prime}$. KELT-23Ab is shown to be above the inflation irradiation threshold $\left(2 \times 10^{8} \mathrm{erg} \mathrm{s}^{-1} \mathrm{~cm}^{-2}\right.$; Demory \& Seager 2011) throughout its history. Bottom: change in semimajor axis of KELT-23Ab over time modeled from several $Q_{*}^{\prime}$ values

Table 6

Transit Times for KELT-23Ab

\begin{tabular}{lccccl}
\hline \hline Epoch & $\begin{array}{c}T_{0} \\
\left(\mathrm{BJD}_{\mathrm{TDB}}\right)\end{array}$ & $\begin{array}{c}\sigma_{T_{0}} \\
(\mathrm{~s})\end{array}$ & $\begin{array}{c}\mathrm{O}-\mathrm{C} \\
(\mathrm{s})\end{array}$ & $\begin{array}{c}\mathrm{O}-\mathrm{C} \\
\left(\sigma_{T_{0}}\right)\end{array}$ & Telescope \\
\hline-3 & 2458144.8984 & 40 & 116 & 2.9 & WCO \\
-3 & 2458144.89724 & 38 & 16 & 0.4 & PvdK \\
1 & 2458153.91793 & 51 & -43 & -0.8 & DEMONEXT \\
0 & 2458153.91681 & 82 & -138 & -1.7 & KeplerCam \\
6 & 2458167.4484 & 95 & -190 & -2.0 & astroLAB \\
15 & 2458187.74583 & 55 & -250 & -4.5 & PvdK \\
20 & 2458196.77035 & 95 & 17 & 0.2 & ASP \\
20 & 2458196.77106 & 54 & 77 & -1.4 & ASP \\
20 & 2458196.7735 & 103 & 285 & 2.7 & WCO \\
54 & 2458273.45249 & 85 & 25 & 0.3 & SOTES \\
66 & 2458302.76997 & 70 & -155 & -2.2 & KUO \\
\hline
\end{tabular}

While KELT-23Ab is not an extreme case of star-planet tidal interactions like WASP-18b (Hellier et al. 2009), the differences in host-star properties, mainly the prevalence of a 
Table 7

Relative Fluxes for the Companion Star, Determined by the SED Analysis (Section 3.2)

\begin{tabular}{lccc}
\hline \hline Filter & $F_{B} / F_{A}$ & Filter & $F_{B} / F_{A}$ \\
\hline$B$ & 0.00134 & $g^{\prime}$ & 0.00146 \\
$V$ & 0.00257 & $r^{\prime}$ & 0.00337 \\
$R$ & 0.00352 & $i^{\prime}$ & 0.00993 \\
$I$ & 0.01282 & $z^{\prime}$ & 0.02166 \\
\hline
\end{tabular}

larger surface convection zone on KELT-23A, could potentially lead to observable TTVs in the system over a long time period. Nonetheless, tidal interactions within the KELT-23Ab system are almost certainly strong enough to conspire with potential Kozai-Lidov oscillations to produce a hot Jupiter, if the nearby star is indeed bound to KELT-23A.

\subsection{Future Observations}

The KELT-23A system presents a unique opportunity for follow up from TESS and the JWST. KELT-23Ab has the second-highest ecliptic latitude $\left(\beta=75^{\circ} .112\right)$ of any transiting exoplanet, second only to Qatar-1b (Alsubai et al. 2011). The system's high ecliptic latitude makes it visible to the JWST $61 \%$ of the year. JWST will be able to characterize planetary atmospheres through the transmission spectroscopy method (Beichman et al. 2014) and KELT-23Ab will be a well-suited candidate for extensive atmospheric studies due to its high equilibrium temperature $\left(T_{\mathrm{eq}}=1561 \mathrm{~K}\right)$ and relatively low surface gravity $\left(\log g_{\mathrm{P}}=3.124\right)$. It is also possible to probe the dayside thermal emission of the planet by obtaining infrared photometry near the secondary eclipse, as described by Rodriguez et al. (2016) in reference to KELT-14b.

The system will also receive multi-sector coverage from $T E S S$, which can be used to determine the transit ephemeris with extremely high precision and detect any TTVs too small to observe from the ground. The photometric precision of TESS would allow for the detection of an Earth-sized companion at an orbital period of several days. TESS will observe many consecutive transits that can be used to study details of the system's transit morphology.

TESS's relatively long observing baseline for KELT-23A will also allow detailed characterization of the planet using the TESS light curve alone. KELT-23A should be observed by TESS in three sectors during year two of its mission. Given KELT-23A's TESS bandpass magnitude of $T=9.739$, we estimate that TESS should achieve a per point photometric precision of $180 \mathrm{ppm} \mathrm{hr}^{-1 / 2}$ on KELT-23A. Over the three 26day sectors, TESS will observe $\sim 34.5$ orbital periods of KELT$23 \mathrm{Ab}$. Phase folding the TESS data on the period implies a binned light-curve precision of $\sim 30 \mathrm{ppm} \mathrm{hr}^{-1 / 2}$. Additionally, the high photometric precision of TESS could detect microvariability of the host star due to stellar oscillations (Campante et al. 2016).

We use the Price \& Rogers (2014) expressions for the analytic precision estimates on the transit observables given finite exposure times to estimate the precision with which TESS can measure the time of transit center $T_{C}$, the ingress/egress durations $\tau$, and the transit and eclipse depths. With the aforementioned per point precision and estimated number of observable transits, TESS should measure $T_{C}$ to $10^{-4}$ days, approximately $9 \mathrm{~s}$, at both the two-minute or thirty-minute cadences (a factor of 10 improvement over the current precision), the ingress/egress duration $\tau$ to fractional uncertainties of $2 \%$ and $6 \%$ at each cadence, and the transit depth $\delta$ to $0.5 \%$ at both cadences. Following Stevens et al. (2018), the improvement in the precision on $\tau$ at the two-minute cadence would improve the stellar density precision to $\approx 3 \%$ (from the current $6 \%$ ) in the limit that the ingress/egress duration is the dominant source of uncertainty.

We calculate the anticipated secondary eclipse depth due to reflected light via Equation (39) of Winn et al. (2010). The depth is $4 \times 10^{-4} A_{\lambda}$, where $A_{\lambda}$ is the geometric albedo. For even a very large albedo, $A_{\lambda}=0.3$, the fractional uncertainties on the reflected light eclipse depths are $71 \%$ and $79 \%$ at the two-minute and thirty-minute cadence.

Assuming that the temperature of KELT-23Ab is equal to the equilibrium temperature, $T_{\mathrm{eq}}=1534 \mathrm{~K}$, as well as perfect heat redistribution in its atmosphere, we calculate the expected secondary eclipse depth from KELT-23Ab's thermal emission to be $5 \mathrm{mmag}$ in the TESS passband; the fractional uncertainty is calculated to be $2 \%$ at both TESS cadences, so thermal emission should be readily detectable.

Using this temperature, the results from our global fit, and Equation (35) of Winn et al. (2010), we calculate a scale height of $3 \times 10^{-3} R_{\mathrm{J}}$ and an expected transmission signal of $10^{-4}$, as is expected for a typical hot Jupiter.

We estimated the amplitudes of three components of the phase curve of KELT-23Ab (Doppler beaming, ellipsoidal variations, and reflection) using the equations presented in Faigler \& Mazeh (2011), Shporer et al. (2011), and Shporer et al. (2019), and our measured parameters of KELT-23Ab. The amplitude of each phase-curve component, however, depends upon a coefficient $\alpha$, which is of order unity (see Equations (1)-3 of Shporer et al. 2011). We assumed that the beaming coefficient $\alpha_{\text {beam }}=1$, and calculated the ellipsoidal and reflection coefficients per Shporer et al. (2019) and Faigler $\&$ Mazeh (2011), respectively, using the linear limb-darkening coefficient from the $i^{\prime}$ band (as an approximation to the TESS bandpass) and estimated albedo, respectively. This resulted in $\alpha_{\text {ellip }}=1.08$, and $\alpha_{\text {refl }}=0.3$ (3.0) if the planetary geometric albedo is $0.03(0.3)$. These imply phase-curve amplitudes of $A_{\text {beam }} \sim 2$ ppm, $A_{\text {ellip }} \sim 3 \mathrm{ppm}$, and $A_{\text {refl }} \sim 11$ (110) ppm. Comparing these to the estimated phase-curve precision, this suggests that the beaming and ellipsoidal components will be undetectable, while the reflected light may be detectable, depending upon the albedo. To the first order, however, the amplitude of the thermal phase curve should be similar to the secondary eclipse depth (assuming a large day-night temperature contrast). Our estimated TESS secondary eclipse depth of 4.9 mmag is much larger than the reflected light component, suggesting that the TESS phase curve will be dominated by thermal emission from the planet.

\section{Conclusion}

KELT-23Ab is an inflated hot Jupiter with an orbital period of 2.255354 days hosted by a G2V solar analog. The starplanet tidal interactions suggest that KELT-23Ab could be consumed by its host in about 1 Gyr. Systems like KELT-23A pose important questions as to why some solar-type stars host hot Jupiters and others do not. TESS will provide many transits that will be used to search for more subtle light-curve effects in the secondary eclipse from the planet's reflection and thermal emission. We calculate that the thermal emission from the planet will be comparable to the secondary eclipse depth and 
will be easily detectable in the TESS phase curve. KELT$23 \mathrm{Ab}$ 's close proximity to the JWST continuous viewing zone means it will be visible for $61 \%$ of the year. This long-term visibility makes KELT-23Ab an excellent follow-up candidate for atmospheric characterization from the JWST.

This project makes use of data from the KELT survey, including support from The Ohio State University, Vanderbilt University, and Lehigh University, along with the KELT follow-up collaboration. Work performed by J.E.R. was supported by the Harvard Future Faculty Leaders Postdoctoral fellowship. D.J.S. and B.S.G. were partially supported by NSF CAREER Grant AST-1056524. Work by S.V.Jr. is supported by the National Science Foundation Graduate Research Fellowship under grant No. DGE-1343012. Work by G.Z. is provided by NASA through Hubble Fellowship grant $H S T$ HF2-51402.001-A awarded by the Space Telescope Science Institute, which is operated by the Association of Universities for Research in Astronomy, Inc., for NASA, under contract NAS 5-26555. This paper includes data taken at The McDonald Observatory of The University of Texas at Austin. This work has made use of NASA's Astrophysics Data System, the Extrasolar Planet Encyclopedia, the NASA Exoplanet Archive, the SIMBAD database operated at CDS, Strasbourg, France, and the VizieR catalog access tool, CDS, Strasbourg, France. We make use of Filtergraph, an online data visualization tool developed at Vanderbilt University through the Vanderbilt Initiative in Data-intensive Astrophysics (VIDA). We also used data products from the Widefield Infrared Survey Explorer, which is a joint project of the University of California, Los Angeles; the Jet Propulsion Laboratory/ California Institute of Technology, which is funded by the National Aeronautics and Space Administration; the Two Micron All Sky Survey, which is a joint project of the University of Massachusetts and the Infrared Processing and Analysis Center/California Institute of Technology, funded by the National Aeronautics and Space Administration and the National Science Foundation; and the European Space Agency (ESA) mission Gaia (http://www.cosmos.esa.int/gaia), processed by the Gaia Data Processing and Analysis Consortium (DPAC, http://www.cosmos.esa.int/web/gaia/ dpac/consortium). Funding for the DPAC has been provided by national institutions, in particular the institutions participating in the Gaia Multilateral Agreement. Any opinions, findings, and conclusions or recommendations expressed are those of the authors and do not necessarily reflect the views of the National Science Foundation. This material is based upon work supported by the National Science Foundation under grant No. 1559487. K.P. acknowledges support from NASA grants 80NSSC18K1009 and NNX17AB94G. We thank Alex Jensen for observations that contributed to this work. J.L. acknowledges support from FAPESP (grant 2017/23731-1). We also thank the anonymous referee for valuable comments that improved this manuscript. D.J. acknowledges support from the Carol and Ray Neag Undergraduate Research Fund.

Software:numpy (Oliphant 2015), matplotlib (Hunter 2007), scipy, (Jones et al. 2001), astropy, (Price-Whelan et al. 2018).

\section{ORCID iDs}

Daniel Johns (10) https://orcid.org/0000-0001-6311-8629

Phillip A. Reed (i) https://orcid.org/0000-0002-5005-1215
Joseph E. Rodriguez (1) https://orcid.org/0000-00018812-0565

Joshua Pepper (1) https://orcid.org/0000-0002-3827-8417

Keivan G. Stassun (1) https://orcid.org/0000-0002-3481-9052

Kaloyan Penev (1) https://orcid.org/0000-0003-4464-1371

B. Scott Gaudi (1) https://orcid.org/0000-0003-0395-9869

Jonathan Labadie-Bartz (i) https://orcid.org/0000-00022919-6786

Benjamin J. Fulton (10 https://orcid.org/0000-0003-3504-5316

Samuel N. Quinn (1) https://orcid.org/0000-0002-8964-8377

Jason D. Eastman (ㄴ) https://orcid.org/0000-0003-3773-5142

Lea Hirsch (1) https://orcid.org/0000-0001-8058-7443

Daniel J. Stevens (1) https://orcid.org/0000-0002-5951-8328

Eric L. N. Jensen (1) https://orcid.org/0000-0002-4625-7333

Steven Villanueva, Jr. (1) https://orcid.org/0000-00016213-8804

Allyson Bieryla (1) https://orcid.org/0000-0001-6637-5401

David W. Latham (1) https://orcid.org/0000-0001-9911-7388

Robert A. Wittenmyer (1) https://orcid.org/0000-00019957-9304

Daniel Bayliss (ํ) https://orcid.org/0000-0001-6023-1335

Thomas G. Beatty (1) https://orcid.org/0000-0002-9539-4203

Karen A. Collins (i) https://orcid.org/0000-0001-6588-9574

Knicole D. Colón (i) https://orcid.org/0000-0001-8020-7121

David James (1) https://orcid.org/0000-0001-5160-4486

Marshall C. Johnson (i) https://orcid.org/0000-0002-

5099-8185

Somayeh Khakpash (1) https://orcid.org/0000-0002-1910-7065

Michael B. Lund (1) https://orcid.org/0000-0003-2527-1598

Mark Manner (1) https://orcid.org/0000-0002-9411-7271

Jennifer L. Marshall (1) https://orcid.org/0000-00030710-9474

Kim K. McLeod (1) https://orcid.org/0000-0001-9504-1486 Matthew T. Penny @il https://orcid.org/0000-0001-7506-5640

Robert J. Siverd (10) https://orcid.org/0000-0001-5016-3359

Chris Stockdale (1) https://orcid.org/0000-0003-2163-1437

Thiam-Guan Tan (10) https://orcid.org/0000-0001-5603-6895

Xinyu Yao (1) https://orcid.org/0000-0003-4554-5592

\section{References}

Agol, E., Steffen, J., Sari, R., \& Clarkson, W. 2005, MNRAS, 359, 567 Alonso, R., Brown, T. M., Torres, G., et al. 2004, ApJL, 613, L153

Alsubai, K. A., Parley, N. R., Bramich, D. M., et al. 2011, MNRAS, 417, 709 Alsubai, K. A., Parley, N. R., Bramich, D. M., et al. 2013, AcA, 63, 465 Bakos, G., Noyes, R. W., Kovács, G., et al. 2004, PASP, 116, 266

Beatty, T. G., Marley, M. S., Gaudi, B. S., et al. 2018, arXiv:1808.09575

Becker, J. C., Vanderburg, A., Adams, F. C., Rappaport, S. A., \& Schwengeler, H. M. 2015, ApJL, 812, L18

Bedell, M., Bean, J. L., Meléndez, J., et al. 2018, ApJ, 865, 68

Beichman, C., Benneke, B., Knutson, H., et al. 2014, PASP, 126, 1134

Bensby, T., Feltzing, S., \& Lundström, I. 2003, A\&A, 410, 527

Bieryla, A., Collins, K., Beatty, T. G., et al. 2015, AJ, 150, 12

Borucki, W. J., Koch, D., Basri, G., et al. 2010, Sci, 327, 977

Brogi, M., de Kok, R. J., Albrecht, S., et al. 2016, ApJ, 817, 106

Buchhave, L. A., Bakos, G. Á., Hartman, J. D., et al. 2010, ApJ, 720, 1118

Buchhave, L. A., Latham, D. W., Johansen, A., et al. 2012, Natur, 486, 375

Butler, R. P., Marcy, G. W., Williams, E., et al. 1996, PASP, 108, 500

Campante, T. L., Schofield, M., Kuszlewicz, J. S., et al. 2016, ApJ, 830, 138

Carter, J. A., \& Winn, J. N. 2009, ApJ, 704, 51

Charbonneau, D., Brown, T. M., Latham, D. W., \& Mayor, M. 2000, ApJL, 529, L45

Charbonneau, D., Brown, T. M., Noyes, R. W., \& Gilliland, R. L. 2002, ApJ, 568,377

Choi, J., Dotter, A., Conroy, C., et al. 2016, ApJ, 823, 102

Ciardi, D. R., Beichman, C. A., Horch, E. P., \& Howell, S. B. 2015, ApJ, 805,16 
Collins, K., \& Kielkopf, J. 2013, AstroImageJ: ImageJ for Astronomy, Astrophysics Source Code Library, ascl:1309.001

Collins, K. A., Collins, K. I., Pepper, J., et al. 2018, AJ, 156, 234

Collins, K. A., Kielkopf, J. F., Stassun, K. G., \& Hessman, F. V. 2017, AJ, 153,77

Coşkunoğlu, B., Ak, S., Bilir, S., et al. 2011, MNRAS, 412, 1237

Coughlin, J. L., Mullally, F., Thompson, S. E., et al. 2016, ApJS, 224, 12

Cutri, R. M., Skrutskie, M. F., van Dyk, S., et al. 2003, yCat, 2246, 0

Cutri, R. M., Wright, E. L., \& Conrow, T. 2012, Explanatory Supplement to the WISE All-Sky Data Release Products, http://wise2.ipac.caltech.edu/ docs/release/allsky/expsup/index.html

Demory, B.-O., \& Seager, S. 2011, ApJS, 197, 12

Dotter, A. 2016, ApJS, 222, 8

Eastman, J. 2017, EXOFASTv2: Generalized publication-quality exoplanet modeling code, Astrophysics Source Code Library, ascl:1710.003

Eastman, J., Gaudi, B. S., \& Agol, E. 2013, PASP, 125, 83

Eastman, J., Siverd, R., \& Gaudi, B. S. 2010, PASP, 122, 935

Fabrycky, D., \& Tremaine, S. 2007, ApJ, 669, 1298

Faigler, S., \& Mazeh, T. 2011, MNRAS, 415, 3921

Fressin, F., Torres, G., Charbonneau, D., et al. 2013, ApJ, 766, 81

Fulton, B. J., Collins, K. A., Gaudi, B. S., et al. 2015, ApJ, 810, 30

Fûrész, G., Szentgyorgyi, A. H., \& Meibom, S. 2008, in Precision Spectroscopy in Astrophysics, ed. N. C. Santos et al. (Berlin: Springer), 287 Furlan, E., Ciardi, D. R., Everett, M. E., et al. 2017, AJ, 153, 71

Gaia Collaboration, Brown, A. G. A., Vallenari, A., et al. 2018, A\&A, 616, A1

Garhart, E., Deming, D., Mandell, A., et al. 2019, arXiv:1901.07040

Gaudi, B. S. 2005, ApJL, 628, L73

Hellier, C., Anderson, D. R., Collier Cameron, A., et al. 2009, Natur, 460, 1098

Hellier, C., Anderson, D. R., Collier Cameron, A., et al. 2012, MNRAS, 426, 739

Henden, A. A., Levine, S., Terrell, D., \& Welch, D. L. 2015, AAS Meeting, $225,336.16$

Henry, G. W., Marcy, G. W., Butler, R. P., \& Vogt, S. S. 2000, ApJL, 529, L41

Høg, E., Fabricius, C., Makarov, V. V., et al. 2000, A\&A, 355, L27

Holman, M. J., \& Murray, N. W. 2005, Sci, 307, 1288

Howell, S. B., Sobeck, C., Haas, M., et al. 2014, PASP, 126, 398

Huang, C. X., Burt, J., Vanderburg, A., et al. 2018, ApJ, 868, L39

Hunter, J. D. 2007, CSE, 9, 90

Jensen, E. 2013, Tapir: A web interface for transit/eclipse observability, Astrophysics Source Code Library, ascl:1306.007

Johnson, M. C., Rodriguez, J. E., Zhou, G., et al. 2018a, AJ, 155, 100

Johnson, M. C., Rodriguez, J. E., Zhou, G., et al. 2018b, AJ, 155, 100

Jones, E., Oliphant, T., Peterson, P., et al. 2001, SciPy: Open source scientific tools for Python, http://www.scipy.org/
Katz, D., Sartoretti, P., Cropper, M., et al. 2019, A\&A, 622, A205

Kovács, G., Zucker, S., \& Mazeh, T. 2002, A\&A, 391, 369

Kurucz, R. L. 1979, ApJS, 40, 1

Kurucz, R. L. 1992, in IAU Symp. 149, The Stellar Populations of Galaxies, ed. B. Barbuy \& A. Renzini (Dordrecht: Kluwer), 225

Labadie-Bartz, J., Rodriguez, J. E., Stassun, K. G., et al. 2019, ApJS, 240, 13

McCullough, P. R., Stys, J. E., Valenti, J. A., et al. 2005, PASP, 117, 783

McLeod, K. K., Rodriguez, J. E., Oelkers, R. J., et al. 2017, AJ, 153, 263

Meléndez, J., Asplund, M., Gustafsson, B., \& Yong, D. 2009, ApJL, 704, L66

Oliphant, T. E. 2015, Guide to NumPy (2nd ed.; Scotts Valley, CA: CreateSpace Independent Publishing Platform)

Paxton, B., Bildsten, L., Dotter, A., et al. 2011, ApJS, 192, 3

Paxton, B., Cantiello, M., Arras, P., et al. 2013, ApJS, 208, 4

Paxton, B., Marchant, P., Schwab, J., et al. 2015, ApJS, 220, 15

Penev, K., Bouma, L. G., Winn, J. N., \& Hartman, J. D. 2018, AJ, 155, 165

Penev, K., Zhang, M., \& Jackson, B. 2014, PASP, 126, 553

Pepper, J., Gould, A., \& Depoy, D. L. 2003, AcA, 53, 213

Pepper, J., Kuhn, R. B., Siverd, R., James, D., \& Stassun, K. 2012, PASP, 124,230

Pepper, J., Pogge, R. W., DePoy, D. L., et al. 2007, PASP, 119, 923

Pollacco, D. L., Skillen, I., Collier Cameron, A., et al. 2006, PASP, 118, 1407

Price, E. M., \& Rogers, L. A. 2014, ApJ, 794, 92

Price-Whelan, A. M., Sipőcz, B. M., Günther, H. M., et al. 2018, AJ, 156, 123

Ricker, G. R., Winn, J. N., Vanderspek, R., et al. 2015, JATIS, 1, 014003

Rodriguez, J. E., Colón, K. D., Stassun, K. G., et al. 2016, AJ, 151, 138

Shporer, A., Jenkins, J. M., Rowe, J. F., et al. 2011, AJ, 142, 195

Shporer, A., Wong, I., Huang, C. X., et al. 2019, AJ, 157, 178

Sing, D. K., Fortney, J. J., Nikolov, N., et al. 2016, Natur, 529, 59

Siverd, R. J., Beatty, T. G., Pepper, J., et al. 2012, ApJ, 761, 123

Stassun, K. G., \& Torres, G. 2018, ApJ, 862, 61

Stefanik, R. P., Latham, D. W., \& Torres, G. 1999, in ASP Conf. Ser. 185, IAU

Colloq. 170, Precise Stellar Radial Velocities, ed. J. B. Hearnshaw \& C. D. Scarfe (San Fransisco, CA: ASP), 354

Steffen, J. H., Ragozzine, D., Fabrycky, D. C., et al. 2012, PNAS, 109, 7982

Stevens, D. J., Gaudi, B. S., \& Stassun, K. G. 2018, ApJ, 862, 53

Szentgyorgyi, A. H., \& Fürész, G. 2007, RMxAA, 28, 129

Talens, G. J. J., Spronck, J. F. P., Lesage, A.-L., et al. 2017, A\&A, 601, A11

Vanderspek, R., Huang, C. X., Vanderburg, A., et al. 2019, ApJL, 871, L24

Vogt, S. S., Radovan, M., Kibrick, R., et al. 2014, PASP, 126, 359

Wheatley, P. J., Pollacco, D. L., Queloz, D., et al. 2013, EPJWC, 47, 13002

Winn, J. N., Fabrycky, D., Albrecht, S., \& Johnson, J. A. 2010, ApJL, 718, L145

Wright, J. T., Marcy, G. W., Howard, A. W., et al. 2012, ApJ, 753, 160 